\title{
STAT3/p53 pathway activation disrupts IFN- $\beta$-induced dormancy in tumor-repopulating cells
}

\author{
Yuying Liu, ${ }^{1,2}$ Jiadi Lv, ${ }^{1}$ Jinyan Liu, ${ }^{1}$ Xiaoyu Liang, ${ }^{1}$ Xun Jin, ${ }^{1}$ Jing Xie, ${ }^{1}$ Le Zhang, ${ }^{1}$ Degao Chen, ${ }^{1}$ Roland Fiskesund, $, 1,3,4$ Ke Tang, ${ }^{5}$ \\ Jingwei Ma, ${ }^{5}$ Huafeng Zhang, ${ }^{5}$ Wenqian Dong, ${ }^{1}$ Siqi Mo, ${ }^{1}$ Tianzhen Zhang, ${ }^{1}$ Feiran Cheng, ${ }^{1}$ Yabo Zhou, ${ }^{1}$ Qingzhu Jia, ${ }^{6}$ Bo Zhu, ${ }^{6}$ \\ Yan Kong, Jun Guo, ${ }^{7}$ Haizeng Zhang, ${ }^{8}$ Zhuo-Wei Hu, ${ }^{9}$ Xuetao Cao, ${ }^{1}$ F. Xiao-Feng Qin, ${ }^{10}$ and Bo Huang ${ }^{1,2,5}$
}

\begin{abstract}
'National Key Laboratory of Medical Molecular Biology and Department of Immunology, Institute of Basic Medical Sciences, and ²Clinical Immunology Center, Chinese Academy of Medical Sciences, Beijing China. ${ }^{3}$ Science for Life Laboratory, Department of Medical Biochemistry and Biophysics, Karolinska Institute, Stockholm, Sweden. ${ }^{4}$ Department of Clinical Immunology and Transfusion Medicine, Karolinska University Hospital, Stockholm, Sweden. ${ }^{5}$ Department of Biochemistry and Molecular Biology, Tongji Medical College, Huazhong University of Science and Technology, Wuhan, China. ${ }^{6}$ Institute of Cancer, Xinqiao Hospital, Third Military Medical University, Chongqing, China. 'Key Laboratory of Carcinogenesis and Translational Research (Ministry of Education), Department of Renal Cancer and Melanoma, Peking University Cancer Hospital and Institute, Beijing, China. ${ }^{8}$ Department of Colorectal Surgery, National Cancer Center/Cancer Hospital, and ${ }^{9}$ Molecular Immunology and Pharmacology Group, State Key Laboratory of Bioactive Substance and Function of Natural Medicines, Institute of Materia Medica, Chinese Academy of Medical Sciences and Peking Union Medical College, Beijing, China. ${ }^{10} \mathrm{Center}$ of Systems Medicine, Institute of Basic Medical Sciences, Chinese Academy of Medical Sciences and Peking Union Medical College, Beijing, and Suzhou Institute of Systems Medicine, Suzhou, China.

Dynamic interaction with the immune system profoundly regulates tumor cell dormancy. However, it is unclear how immunological cues trigger cancer cell-intrinsic signaling pathways for entering into dormancy. Here, we show that IFN- $\beta$ treatment induced tumor-repopulating cells (TRC) to enter dormancy through an indolamine 2,3-dioxygenase/kynurenine/ aryl hydrocarbon receptor/p27-dependent (IDO/Kyn/AhR/p27-dependent) pathway. Strategies to block this metabolic circuitry did not relieve dormancy, but led to apoptosis of dormant TRCs in murine and human melanoma models. Specifically, blocking AhR redirected IFN- $\beta$ signaling to STAT3 phosphorylation through both tyrosine and serine sites, which subsequently facilitated STAT3 nuclear translocation and subsequent binding to the $\mathrm{p} 53$ promoter in the nucleus. Upregulation of $p 53$ in turn disrupted the pentose phosphate pathway, leading to excessive ROS production and dormant TRC death. Additionally, in melanoma patients, high expression of IFN- $\beta$ correlated with tumor cell dormancy. Identification of this mechanism for controlling TRC dormancy by IFN- $\beta$ provides deeper insights into cancer-immune interaction and potential new cancer immunotherapeutic modalities.
\end{abstract}

\section{Introduction}

Various types of viruses are capable of infecting the host, causing viral persistence, latency, and host tumorigenesis, typically including liver, cervical, and nasopharyngeal cancers and some subtypes of leukemia. However, how the host mobilizes both innate and adaptive antiviral arms to prevent viral-induced tumorigenesis and maintain intrinsic homeostasis has only begun to be understood, especially for type I IFNs, a key innate molecule of host defense against viral infections, which may determine and/or shape viral infection-caused tumorigenesis. The type I IFN receptor is ubiquitously expressed by host cells, including tumor cells. Intriguingly, a host defect related to type I IFNs or their signaling can result in different cell-type transformations and tumorigenesis (1-4). In fact, type I IFNs are known to effectively activate DC maturation and are required for the early stage priming of tumor-specific $\mathrm{T}$ cells $(5,6)$. Thus, as a typical innate antiviral arm, type I IFNs seem to possess intrinsic antitumor abilities. However, the precise role of type I IFNs in mediating antitumor immunity still remains largely unclear.

Authorship note: Y. Liu, J. Lv, and J.Liu contributed equally to this work. Conflict of interest: The authors have declared that no conflict of interest exists. Submitted: July 19, 2017; Accepted: January 2, 2018.

Reference information: / Clin Invest. 2018;128(3):1057-1073.

https://doi.org/10.1172/JCI96329.
The consequence of a host-virus interaction is usually either death of the infected cell or disruption of the virus. However, for certain viral pathogens, cells neither clear them quickly nor are killed by them. In this case, it is reasonable to speculate that cells might use another strategy to counteract such sublethal invaders, by entering dormancy and waking up after the spontaneous decay of the invader inside. However, the kind of signal or signals that initiate these infected cells to enter dormancy remains unclear. Ancestral cells evolved the IFN- $\alpha / \beta$ pathway to defend against viral invasions, and it is possible that modern cells inherit type I IFNs as a common means to initiate the dormancy defense process against invading pathogenic viruses. Coincidently, type I IFNs are known to have antiproliferative activity and can mediate cellcycle arrest $(7,8)$, an important feature of cellular dormancy. Based on this knowledge, we hypothesized that type I IFNs have the capacity to control tumor cells by inducing them into dormancy.

Clinical observations from transplantations suggest that the immune system is capable of inducing tumor dormancy (9-11), and metastasizing breast tumors have been found to survive at the bone marrow site, a central immune organ, for decades without growth (12). However, how our body's immune responses participate in the entry of tumor cells into dormancy remains unknown. Stem cell-like cancer cells (SCLCC) are a self-renewing, highly tumorigenic subset of cancer cells with a crucial role in 
tumorigenesis, metastasis, and drug resistance (13-15). Whether SCLCCs can more readily enter dormancy by immune cues also remains unclear. We recently developed a mechanics-based method to select and amplify a minor population of tumor cells by culturing single tumor cells in soft 3D fibrin gels (13-17). The growing colonies showed spheroid-like morphological changes. More importantly, when these spheroids were digested into single cells by dispase, as few as 10 selected cells were able to grow tumors in immunocompetent mice (13). We thus functionally defined these soft 3D fibrin gel-cultured cells as tumor-repopulating cells (TRC). In this study, we demonstrate that IFN- $\beta$ treatment induces melanoma TRCs into dormancy through an indolamine 2,3-dioxygenase/aryl hydrocarbon receptor/27-dependent (IDO1/AhR/p27-dependent) pathway and that disrupting this pathway by blocking IDO1 or AhR can redirect IFN- $\beta$ signaling through a STAT3/p53/ROS-mediated pathway, leading to apoptotic cell death of dormant TRCs.

\section{Results}

IFN- $\beta$ treatment induces stem cell-like melanoma cells into dormancy in vivo. Type I IFN is one of the approved drugs for melanoma treatment $(18,19)$. In addition to its immunomodulatory effect, type I IFN is also reported to exert direct inhibitory effects on melanoma cells $(20,21)$. In order to better understand the effect of type I IFN on melanoma cells in vivo, we treated C57BL/6 mice bearing s.c. implanted B16 melanomas of $5 \times 5 \mathrm{~mm}$ in size with intratumoral injection of IFN- $\beta$ ( $250 \mathrm{ng} / \mathrm{d})$ for 3 days and then isolated single tumor cells. Cell-cycle analysis showed that IFN- $\beta$ treatment markedly increased the $G_{0} / G_{1}$ proportion in the living tumor cells from $20.5 \%$ to $72.4 \%$ (Figure $1 \mathrm{~A}$ ). It is known that quiescent and senescent cells both have a $G_{0} / G_{1}$ arrest state. When we used $\beta$-gal staining to determine senescence, the above melanoma cells did not show blue staining, indicating that the cells did not enter senescence (Figure 1B). Dormancy is another type of $\mathrm{G}_{0} / \mathrm{G}_{1}$ arrest state and is often related to undifferentiated stem cells with downregulated metabolic process and protein translation (22-25). CD133 is commonly used to identify SCLCCs in tumors, including melanomas $(26,27)$. Here, we sorted CD133 $3^{\text {hi }}$ tumor cells from B16 melanoma. We found that IFN- $\beta$ treatment did not reduce the number of $\mathrm{CD} 133^{\text {hi }} \mathrm{B} 16$ cells in tumor mass (Figure $1 \mathrm{C}$ ), but resulted in their entering $\mathrm{G}_{0} / \mathrm{G}_{1}$ cell-cycle arrest (Figure 1D). However, IFN- $\beta$ treatment did not induce $\mathrm{G}_{0} / \mathrm{G}_{1}$ cell-cycle arrest of CD133- $\mathrm{B} 16$ cells (Supplemental Figure 1A; supplemental material available online with this article; https://doi.org/10.1172/JCI96329DS1). Moreover, immunostaining with CD133 antibody and Ki67 (a cell-proliferating marker) showed that IFN- $\beta$ treatment resulted in much less Ki67 staining in $\mathrm{CD} 133^{\text {hi }}$ tumor cells compared with the control group (Supplemental Figure 1B). In addition to murine B16 cells, we also s.c. injected human A375 melanoma cells into NOD-SCID mice. When the tumors reached $5 \times 5 \mathrm{~mm}$, these mice were treated with IFN- $\beta$ ( $250 \mathrm{ng} / \mathrm{d}$ ) for 3 days. Consistently, IFN- $\beta$ treatment induced $A 375$ into $G_{0} / G_{1}$ arrest, but did not exhibit the sign of senescence (Supplemental Figure 1, C and D). We then further examined whether the treated tumor entered a dormant state by IFN- $\beta$. To this end, C57BL/6 mice were injected s.c. with $5 \times 10^{3} \mathrm{CD} 133^{+}$B16 TRCs for 3 days and then treated with IFN- $\beta$ $(50 \mathrm{ng} / \mathrm{d})$. On day 5 , a tiny melanoma nest at the injection site was confirmed by immunohistochemical and H\&E staining. On day 20 , melanoma in the PBS control group had grown to be $5 \times 5 \mathrm{~mm}$, while IFN- $\beta$ treatment kept the original tumor size as observed on day 5 , suggesting that IFN- $\beta$ treatment induces a growth arrest state of melanoma in vivo (Figure $1 \mathrm{E}$ ). However, the administration of IFN- $\beta$-neutralizing antibody beginning at day 10 resulted in the regrowth of the tumor in the IFN- $\beta$-treated mice (Figure $1 F)$, suggesting that IFN- $\beta$ treatment indeed leads to a reversible dormant state of melanoma. Similar results were obtained when NOD-SCID mice were injected s.c. with $1 \times 10^{4}$ A375 melanoma TRCs and treated with or without IFN- $\beta$ (75 ng per d) in the presence or absence of anti-IFN- $\beta$ antibody (Supplemental Figure 1, $\mathrm{E}$ and $\mathrm{F})$. In addition, we immunostained the above tissue with the dormant markers (NR2F1/Ki67 or DEC2/Ki67) as reported $(28,29)$ and found that IFN- $\beta$ treatment remarkably increased the percentage of $\mathrm{NR}^{2} \mathrm{~F}^{+}{ }^{+} \mathrm{Ki} 67^{-}$cells (Figure $1 \mathrm{G}$ ) or $\mathrm{DEC}^{+} \mathrm{Ki} 67^{-}$ cells (Supplemental Figure 1G), further confirming that IFN- $\beta$ treated melanoma cells are in a dormant state. Together, these data suggest that IFN- $\beta$ treatment is capable of inducing stem-like melanoma cells into dormancy in vivo.

IFN- $\beta$ induces melanoma TRCs into dormancy in vitro. Next, we tried to validate the above in vivo data in vitro. Despite the importance of stem cell-like tumor cells in tumor initiation, progression, metastasis, and drug resistance, a hindrance lies in that this population belongs to a minor subpopulation and that the number insufficiency restricts extensive mechanistic study on stem cell-like tumor cells. To overcome this limitation, we previously established a mechanics-based 3D soft fibrin gel culture system to select and amplify TRCs (13-16). When we seeded $\mathrm{CD} 133^{\text {hi }}$ B16 or A375 stem-like melanoma cells into the soft fibrin gels, we found that most of the cells could grow into colonies (Supplemental Figure 2A). In contrast, less than $8 \%$ of CD133- B16 cells could grow into colonies in the soft 3D fibrin gels, consistent with our previous report (30), suggesting that CD133 ${ }^{\text {hi }}$ melanoma cells represent TRCs. Thus, in the following studies, we used in vitro culture-enriched and expanded melanoma TRCs to investigate the mechanistic aspects of how IFN- $\beta$ induces stem-like melanoma cells into dormancy. In line with our in vivo data, we found that, although B16 TRCs grew rapidly in soft $3 \mathrm{D}$ fibrin gels, addition of IFN- $\beta$ significantly inhibited their growth in a dose-dependent manner and that 5 $\mathrm{ng} / \mathrm{ml}$ of IFN- $\beta$ could completely block B16 or A375 TRC proliferation (Figure 2A). The cell-cycle analysis showed significant $\mathrm{G}_{0} / \mathrm{G}_{1}$ arrest in both TRCs (Figure $2 \mathrm{~B}$ ); however, these quiescent TRCs could start to regrow upon IFN- $\beta$ removal (Figure 2A), suggesting that IFN- $\beta$ possibly induces dormancy in melanoma TRCs. Indeed, we found that IFN- $\beta$ treatment resulted in more than $90 \%$ TRCs having a $\mathrm{NR}^{2} \mathrm{~F}^{+} \mathrm{Ki}^{-}$or $\mathrm{DEC}^{+} \mathrm{Ki}^{-}$dormant phenotype (Figure 2C). Apart from demonstrating $G_{0} / G_{1}$ cell-cycle arrest in TRCs, we also found that B16 and A375 TRCs decreased glucose consumption in the presence of IFN- $\beta$ (Figure 2D). In addition, IFN- $\beta$ did not induce B16 and A375 TRCs to undergo senesence, as evaluated by $\beta$-gal activity (Figure 2E). Dormant tumor cells may also decrease their response to xenobiotics, including chemotherapeutic drugs $(31,32)$. We found that IFN- $\beta$-treated B16 and A375 TRCs were more resistant to methatrexate and paclitaxol than control TRCs (Figure 2F). Not- 
A

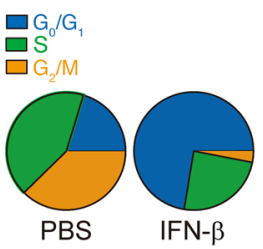

B

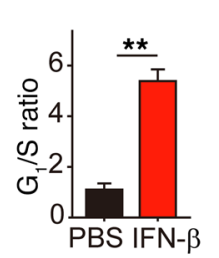

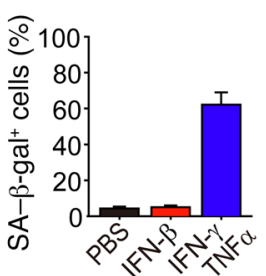

C

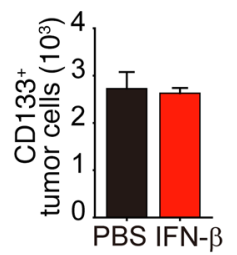

D

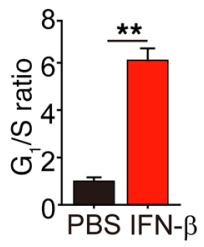

E
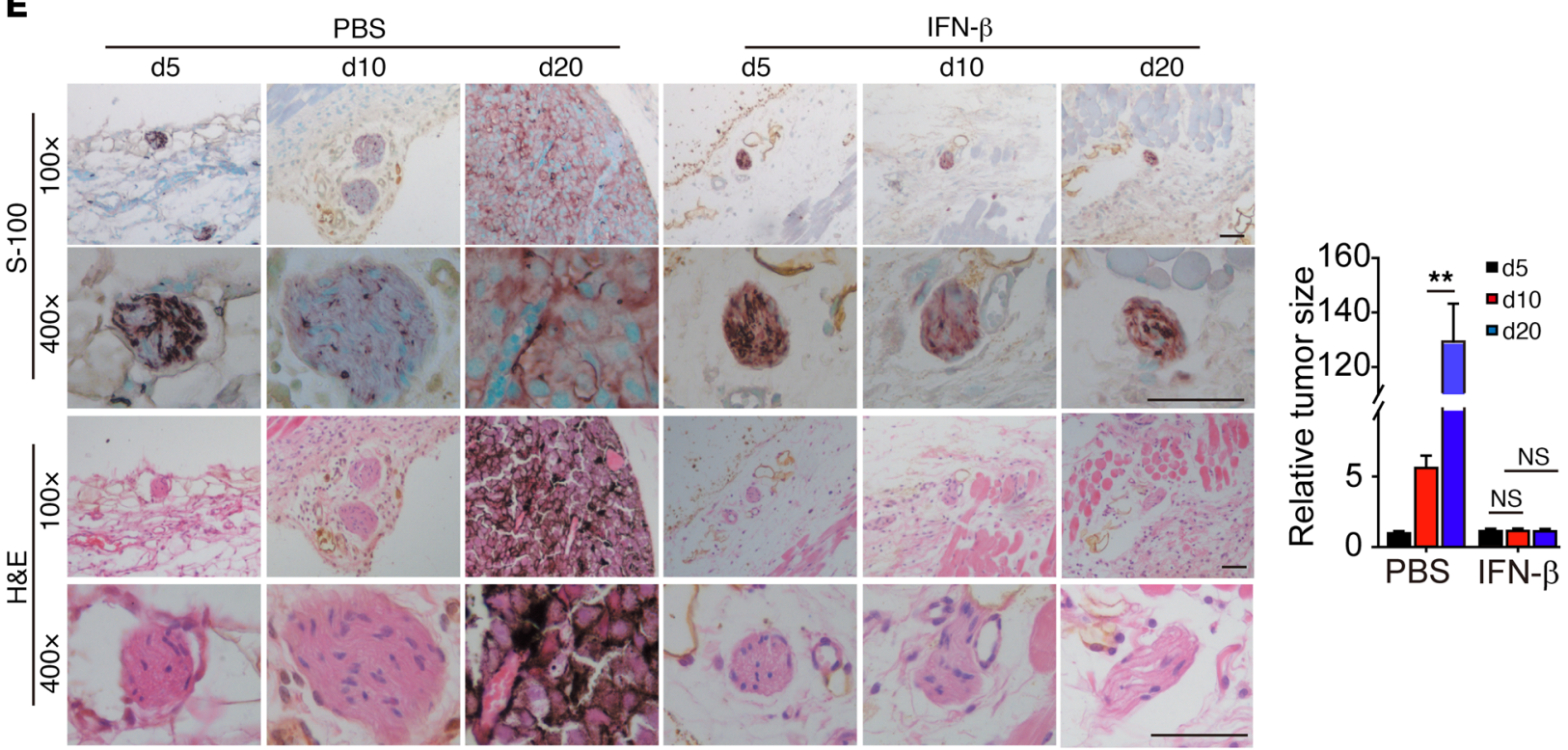

$\mathbf{F}$

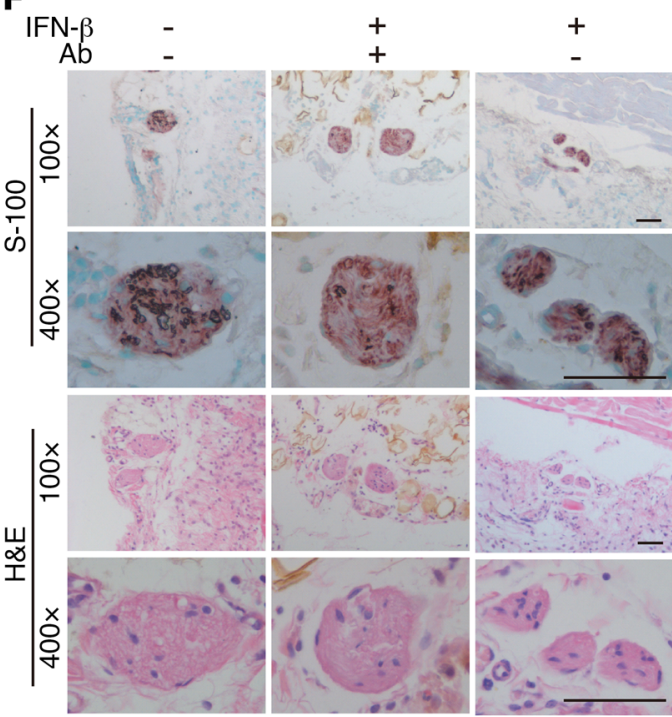

G
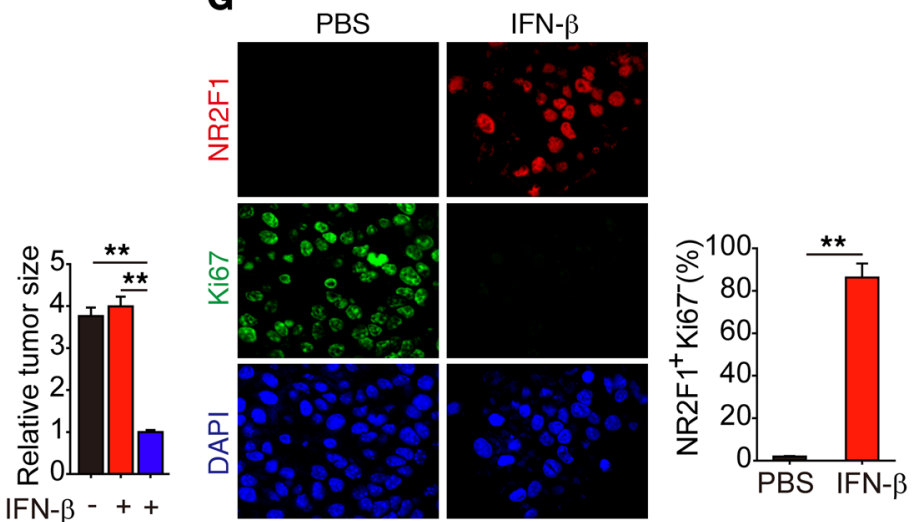

$\mathrm{FN}-\beta-++$
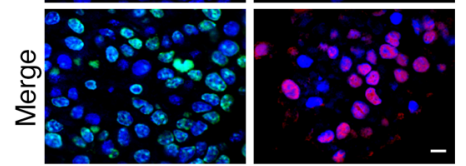

Figure 1. IFN- $\beta$ induces TRC dormancy in vivo. (A) C57BL/ 6 mice with $5 \times 5 \mathrm{~mm}$ B16 melanoma were intratumorally treated with $250 \mathrm{ng}$ IFN- $\beta$ once per day for 3 days. Isolated tumor cells were assayed for cell-cycle analysis $(n=5)$. (B) As in A, but some mice were treated with $10 \mu \mathrm{g}$ IFN- $\gamma+$ TNF- $\alpha$ for 3 days as positive control. Isolated tumor cells were stained with SA- $\beta$-gal $(n=5)$. (C and $\mathbf{D})$ As in $\mathbf{A}$, but CD133 ${ }^{\text {hi }}$ tumor cells were counted by flow cytometry (C) $(n=5)$, and the cell cycle of CD133 ${ }^{\text {hi }}$ tumor cells was analyzed (D) $(n=5)$. (E) B16 TRCs $\left(5 \times 10^{3}\right)$ were s.c. injected into mice. On day $3,50 \mathrm{ng}$ IFN- $\beta$ was injected into the tumor site once every 2 days. On days 5, 10, and 20, tumor cell-injected tissues were analyzed by immunostaining against S100 $\beta$ or $H \& E$ staining. Tumor size is presented photographically (left) and graphically (right) $(n=6)$. Scale bars: $50 \mu \mathrm{m}$. (F) Mice subcutaneously injected with $5 \times 10^{3}$ B16 TRCs were intratumorally treated with IFN- $\beta(50 \mathrm{ng} / \mathrm{d}$ ) for 10 days and then further treated with IFN- $\beta$ or IFN- $\beta+$ anti-IFN- $\beta$ antibody once every 2 days for 5 days. Tissues at the injection site were used for immunostaining for $S 100 \beta$ and stained with $\mathrm{H} \& \mathrm{E}(n=6)$. Scale bars: $50 \mu \mathrm{m}$. (C) The same as $\mathbf{E}$, except that at day 20, tissues with tumor cell inoculation were immunostained with anti-NR2F1, -Ki67, and DAPI $(n=5)$. Scale bar: $10 \mu \mathrm{m}$. Data represent mean \pm SEM. ${ }^{* *} P<0.01,2$-tailed Student's $t$ test $(\mathbf{A}, \mathbf{D}$, and $\mathbf{G})$ and 1-way ANOVA (E and $\left.\mathbf{F}\right)$. 
A

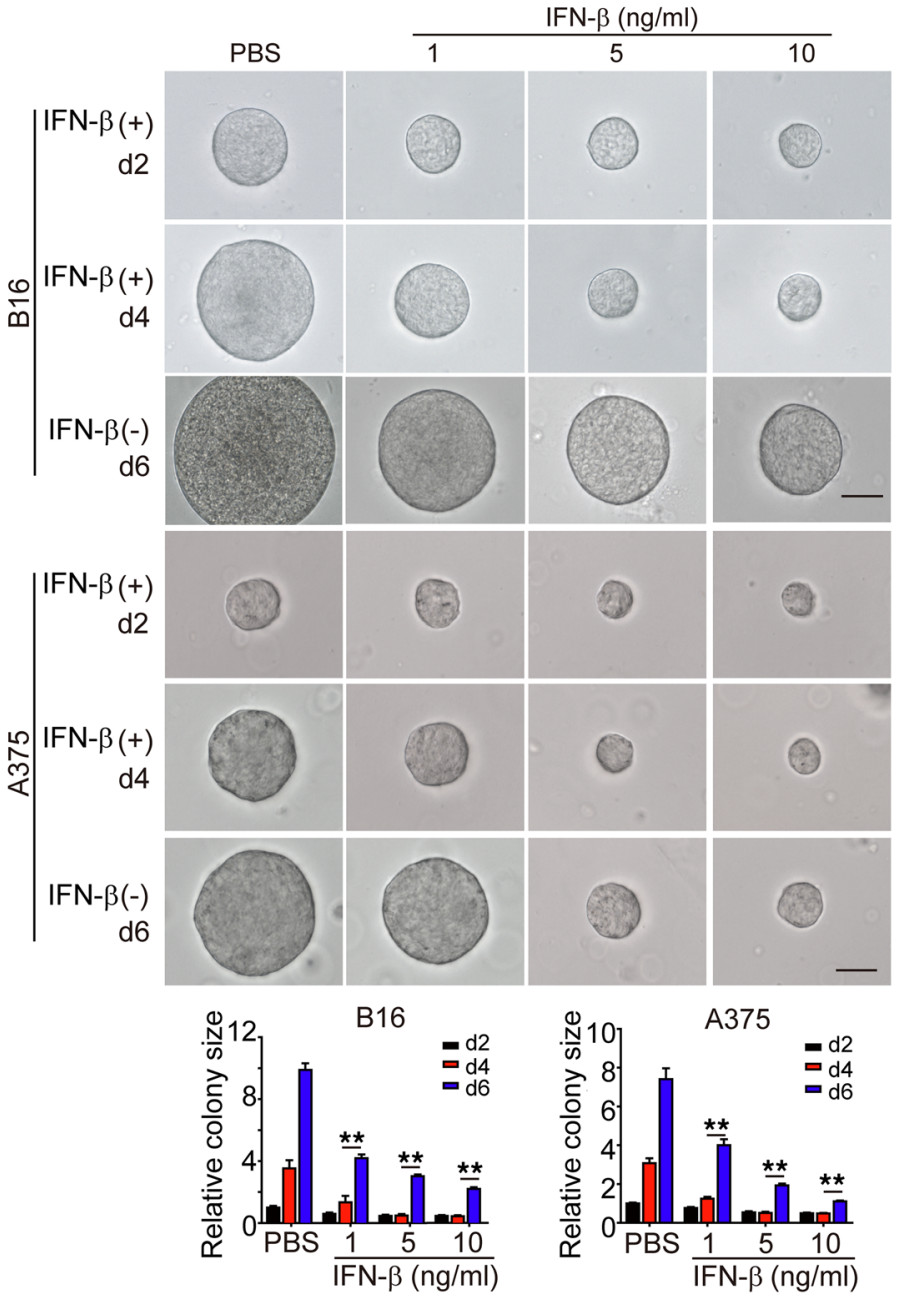

B
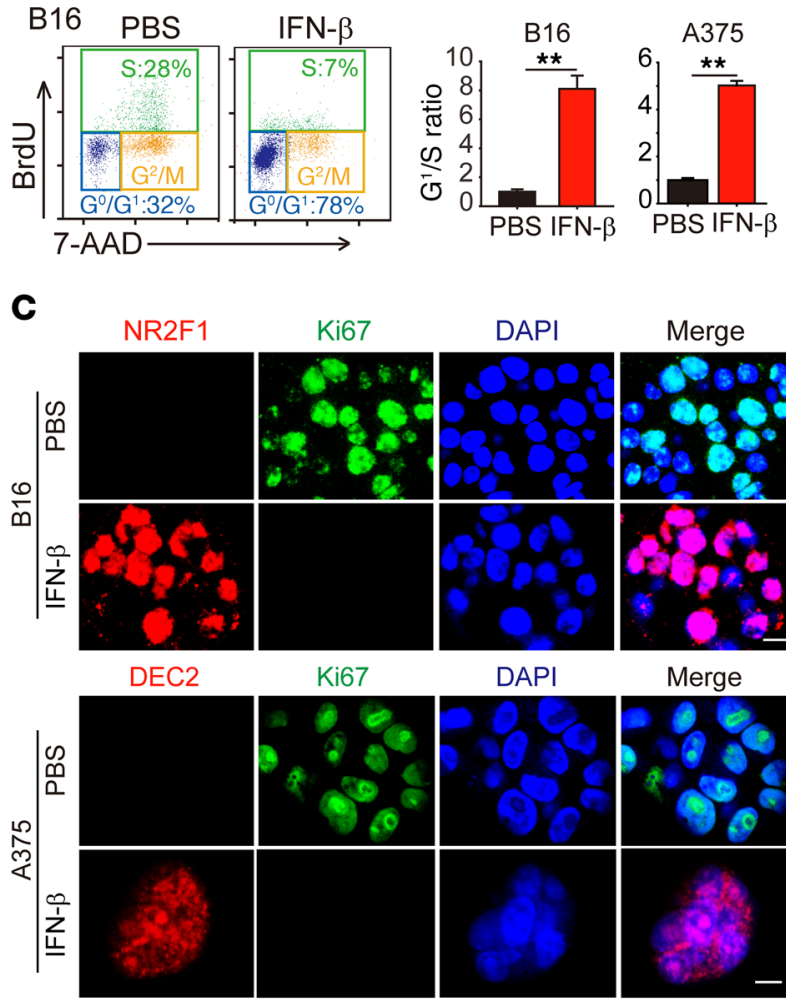

Merge

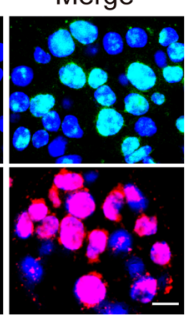

DAPI
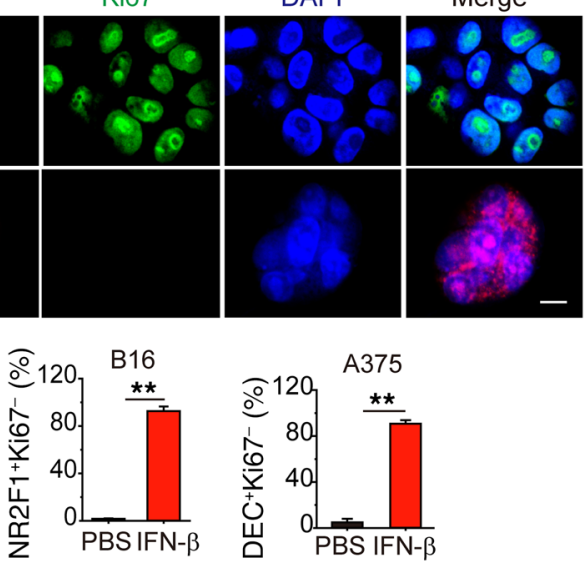
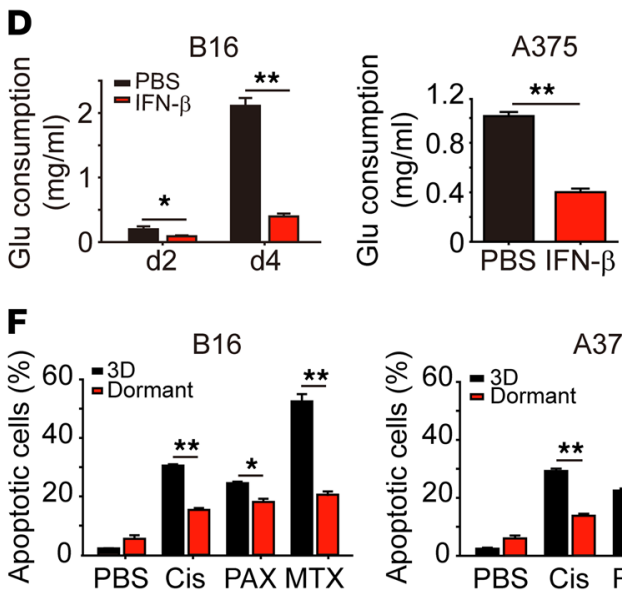

E
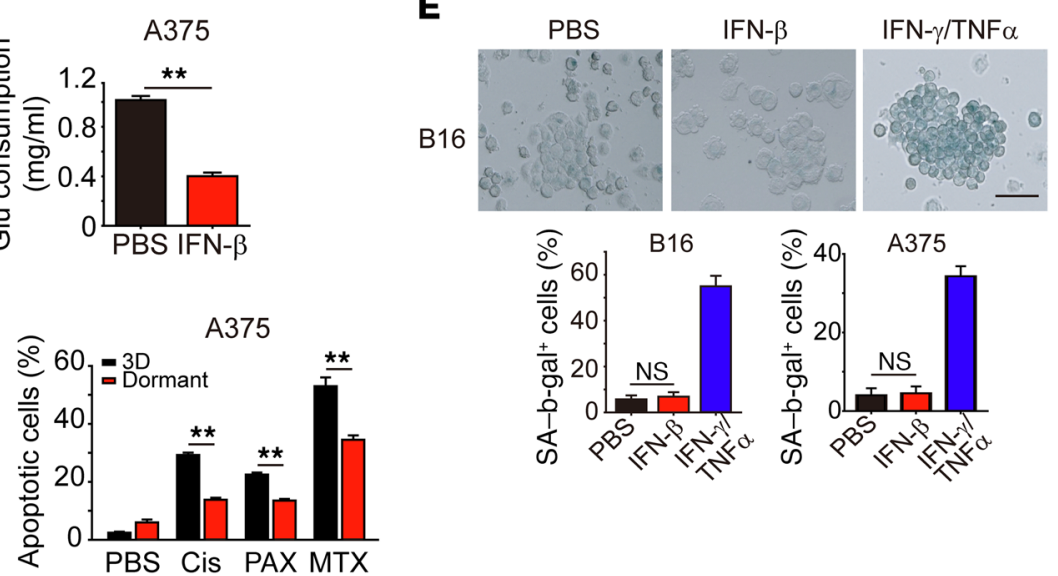

Figure 2. IFN- $\boldsymbol{\beta}$ induces TRC dormancy in vitro. (A and B) B16 or A375 TRCs seeded in soft 3D fibrin gels were cultured for 2 days and then treated with different doses of IFN- $\beta$ for an additional 2 days (d2) or 4 days. In another setting, IFN- $\beta$ was removed from day 4 and the colonies were measured on day 6. Colony size was indicated (A), and cell cycle was analyzed after 3 days of IFN- $\beta$ treatment ( $5 \mathrm{ng} / \mathrm{ml}$ ) (B). Scale bars: $50 \mu \mathrm{m}$. (C) The same as B, but immunostaining of NR2F1/Ki67 or DEC2/Ki67 was performed. Scale bars: $10 \mu \mathrm{m}$. (D) IFN- $\beta$ treatment decreased glucose (Clu) consumption in B16 or A375 TRCs. (E) B16 or A375 TRCs were treated with IFN- $\beta$ or IFN- $\gamma /$ TNF- $\alpha$ for 72 hours. SA- $\beta$-gal staining was conducted. (F) B16 TRCs, A375 TRCs, or dormant B16 or A375 TRCs (48 hours IFN- $\beta$ treatment) were treated with cisplatin (Cis) ( $3 \mu \mathrm{g} / \mathrm{ml})$, MTX ( $2 \mu \mathrm{g} / \mathrm{ml})$, or PAX ( $3 \mu \mathrm{g} / \mathrm{ml})$ for 24 hours. Cell viability was measured by flow cytometry. Graphs represent mean \pm SEM of 3 independent experiments. ${ }^{*} P<0.05 ;{ }^{*} P<0.01,1$-way ANOVA (A and E) and 2-tailed Student's $t$ test (B-D and F). 
withstanding the dormancy induction on TRCs, IFN- $\beta$ was not able to induce the dormancy of differentiated B16 cells cultured in rigid plastic (Supplemental Figure 2, B and C). Together, these data suggest that IFN- $\beta$ is capable of inducing melanoma TRCs into dormancy in vitro.

IFN- $\beta$ activated IDO1/Kyn/AhR/p27 to mediate melanoma TRC dormancy. IFN- $\beta$ binds its receptor IFNAR1/IFNAR2 and activates STAT1/STAT2 for the transduction of IFN- $\beta$ signaling (8). To investigate the molecular mechanism involved in IFN- $\beta$-induced TRC dormancy, we knocked down STAT1 and STAT2, respectively, and found that knockdown of either STAT1 or STAT2 resulted in the failure of TRCs to become dormant in the presence of IFN- $\beta$ (Supplemental Figure 3, A and B). Intriguingly, we found that active IFN- $\alpha$ at different concentrations (1, $5,10,100$, and $200 \mathrm{ng} / \mathrm{ml}$ ) did not induce TRCs into dormancy (Supplemental Figure 3, C and D), suggesting that selective activation of STAT1 and STAT2 by IFN- $\beta$ is required for TRC dormancy. IDO1, an enzyme that catalyzes tryptophan metabolism, is well known to be upregulated by IFN- $\gamma /$ STAT1 signaling (33, 34). Indeed, real-time PCR and Western blot showed that IDO1 was markedly upregulated in IFN- $\beta$-treated B16 or A375 TRCs (Figure 3A and Supplemental Figure 3E). Based on these data, we postulated that IDO1 was involved in IFN- $\beta$-induced TRC dormancy. In support of this assumption, kynurenine (Kyn), a major metabolic intermediate of IDO1-catalyzed tryptophan, was strikingly upregulated in IFN- $\beta$-treated B16 or A375 TRCs (Figure $3 \mathrm{~B}$ ). Kyn is known to be an endogenous ligand for AhR (35), a critical cytoplasmic transcription factor, which translocates into the nucleus upon activation and regulates gene expression through binding to a specific DNA sequence called dioxin-responsive element (DRE) (36). In line with the increased level of Kyn, enhanced transcription activity of AhR in IFN- $\beta$ treated B16 or A375 TRCs was observed, as demonstrated by the DRE-luciferase assay (Figure 3C). Additionally, both immunostaining assay and Western blot analysis showed that a majority of AhR protein molecules had translocated from the cytoplasm to the nucleus upon IFN- $\beta$ or Kyn treatment (Figure 3, D and E), suggesting that AhR might be involved in the downstream process of IFN- $\beta$-induced TRC dormancy. Given that cell-cycle arrest is a typical feature for cellular dormancy and that the p27 promoter region contains 3 DRE core sequences (5'-CACGCNA-3'), we explored the possibility of AhRregulating cell cycle inhibitor gene(s) in IFN- $\beta$-treated TRCs. First, we found that, like IDO1 and AhR, p27 was also upregulated in IFN- $\beta$-treated TRCs (Figure 3F and Supplemental Figure $3 \mathrm{~F})$. By cotransfecting mouse and human $\mathrm{p} 27$ promoter-reporter (pp27-Luc) and Flag-AhR into B16 or A375 TRCs, we observed a 20-fold increase of luciferase activity in B16 TRCs and an 8-fold increase in A375 TRCs upon IFN- $\beta$ treatment (Figure 3G), suggesting that IFN- $\beta$ signaling activates $A h R$ to upregulate the expression of $\mathrm{p} 27$. Moreover, we found that $p 27$ knockout could break IFN- $\beta$-induced dormancy of B16 or A375 TRCs (Figure $3 \mathrm{H}$ and Supplemental Figure $3 \mathrm{G}$ ). In addition, in p27-, IDO1-, or $A h R$-knockout B16 TRCs, IFN- $\beta$ treatment $(5 \mathrm{ng} / \mathrm{ml})$ was not able to induce an NR2F1+Ki67- dormant phenotype (Supplemental Figure 3H). Together, these data indicate that the AhR/p27 pathway mediates IFN- $\beta$-induced TRC dormancy.
Blockade of AhR switches IFN- $\beta$-induced TRC dormancy to apoptosis. Based on the data showing that IFN- $\beta$ uses the AhRdependent pathway to induce melanoma in TRC dormancy, we speculated that blockade of AhR could break dormancy and lead to the regrowth of TRCs. To our surprise, dormant B16 or A375 TRCs did not regrow upon pharmacological AhR blockade, but instead underwent apoptosis, as evidenced by decreased colony numbers and colony sizes of TRCs in soft 3D fibrin gels (Figure $4, \mathrm{~A}$ and B). In addition, knocking out $A h R$ also led to decreases in colony size and number (Supplemental Figure 4, A and B). Flow cytometric analysis further confirmed that IFN- $\beta$ treatment plus AhR blockade induced apoptosis in dormant TRCs (Figure 4C). Since IDO1 can activate AhR through Kyn production by converting tryptophan, we repeated the above-mentioned experiments with the IDO1 inhibitor 1-methyl-D-tryptophan (1-MT). As expected, the addition of 1-MT also reduced colony size and the number of TRCs in the colony (Figure 4, D and E), which was further confirmed by IDO1 knockdown (Supplemental Figure 4, C and D) and flow cytometric analysis (Figure $4 \mathrm{~F}$ ). Intriguingly, although 1-MT induced apoptosis of IFN- $\beta$-treated TRCs, such apoptosis could be rescued by the addition of Kyn (Figure $4 \mathrm{G}$ ), further confirming that IFN- $\beta$ uses IDO1/Kyn/AhR to mediate TRC dormancy and that blockade AhR may cause IFN- $\beta$-induced TRC apoptosis.

Nuclear translocation of serine- but not tyrosine-phosphorylated STAT3 mediates IFN- $\beta / A h R$ inhibitor-induced TRC apoptosis. Next, we tried to elucidate the mechanism through which AhR blockade caused apoptosis in IFN- $\beta$-treated TRCs. Besides STAT1 and STAT2, IFN- $\beta$ signaling can also activate STAT3 (37-39). Intriguingly, STAT3 in turn can regulate IFN- $\beta$ signaling (40). Moreover, STAT3 is considered an oncogene that profoundly promotes tumorigenesis $(41,42)$. We therefore assumed that the IFN- $\beta / A h R$ inhibitor exerts its effect through STAT3 to cause dormant TRC apoptosis. To this end, we knocked out STAT3 in TRCs (Supplemental Figure $5 \mathrm{~A})$. As a result, we found that STAT3 knockout saved IFN- $\beta$-treated TRCs from apoptosis induced by dimethoxyflavone (DMF) or 1-MT (Figure $5 \mathrm{~A}$ ) and that these dormant TRCs could wake up and start to grow as well as lose the dormant marker NR2F1+Ki67- (Supplemental Figure 5, B and C), suggesting that STAT3 indeed mediates the above-described TRC dormancy abrogation. In addition, we found that high-dose IFN- $\beta$ (10 ng/ml), but not low dose IFN- $\beta(2.5 \mathrm{ng} / \mathrm{ml})$, was able to induce STAT3 knockout B16 TRCs into dormancy, while a low dose of IFN- $\beta$ could induce WT B16 TRCs into dormancy (Supplemental Figure 6, A and B), implying that IFN- $\beta$-induced AhR plays an important role in TRC dormancy. Then we explored the STAT3involved molecular events. We found that tyrosine (Y) phosphorylation, considered as a marker for STAT3 activation, although being highly expressed in B16 TRCs, was markedly decreased upon IFN- $\beta$ treatment (Figure 5B). However, this IFN- $\beta$-induced decrease of tyrosine phosphorylation could be recovered by blockade of AhR activity by either DMF- or AhR-targeting single guide RNAs (sgRNAs) (Figure 5B and Supplemental Figure 6C), suggesting that AhR activation may downregulate STAT3 tyrosine phosphorylation. In support of this notion, the addition of Kyn or overexpression of IDO1 to activate AhR led to TRCs reducing phosphorylated STAT3 (p-STAT3) (Y) (Figure 5C). Then, we further investigated the regulation of AhR on tyrosine phosphor- 

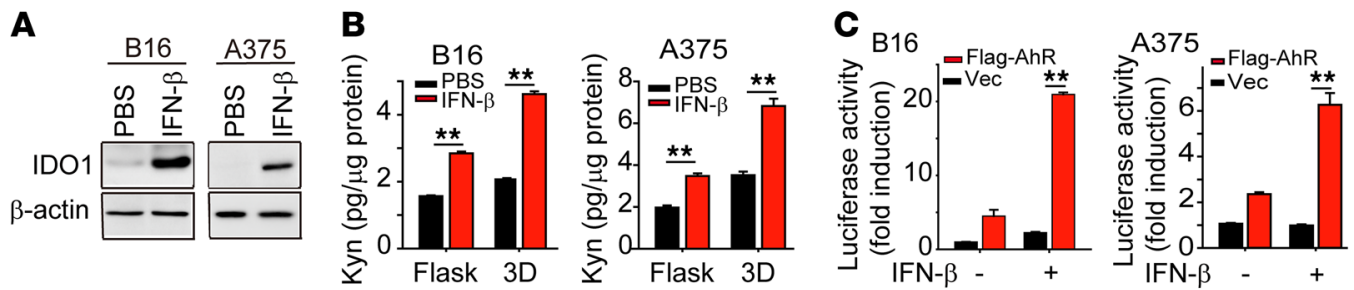
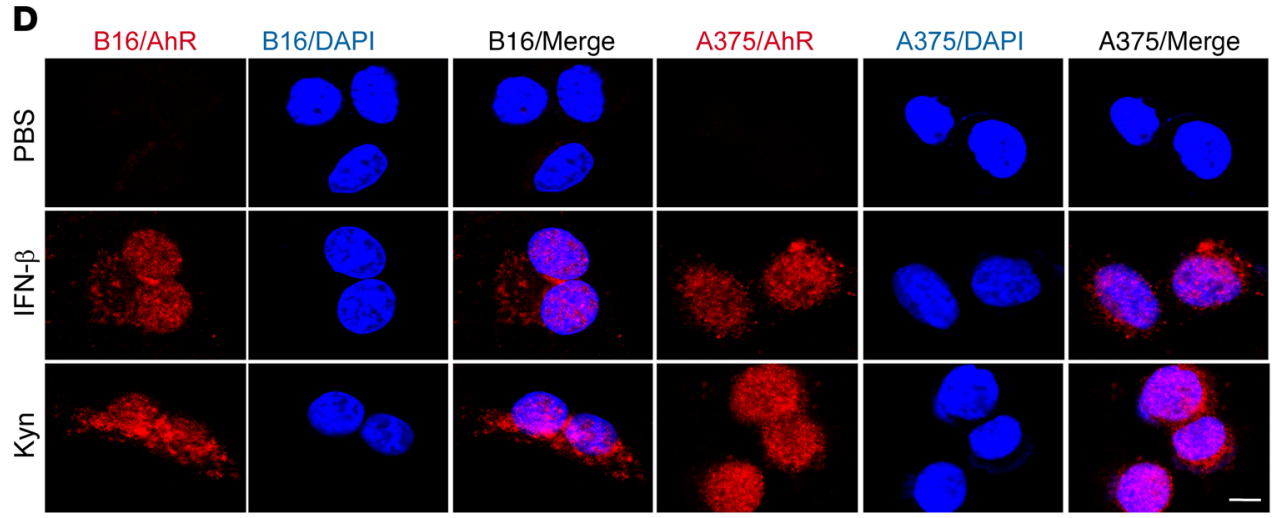

E

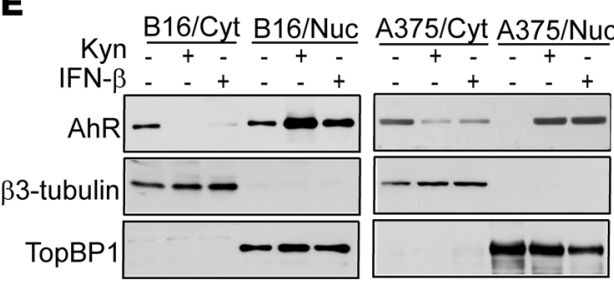

F

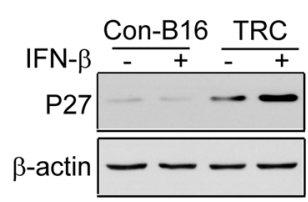

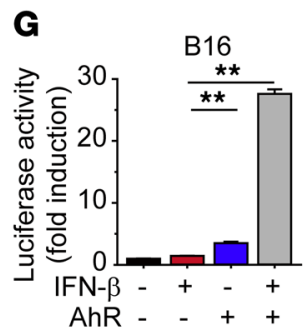

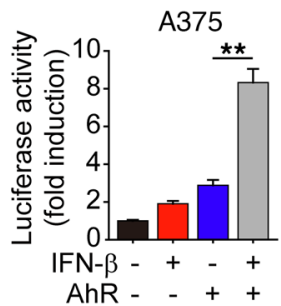

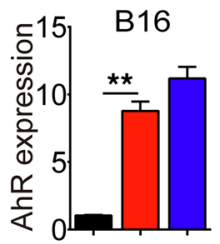

A375
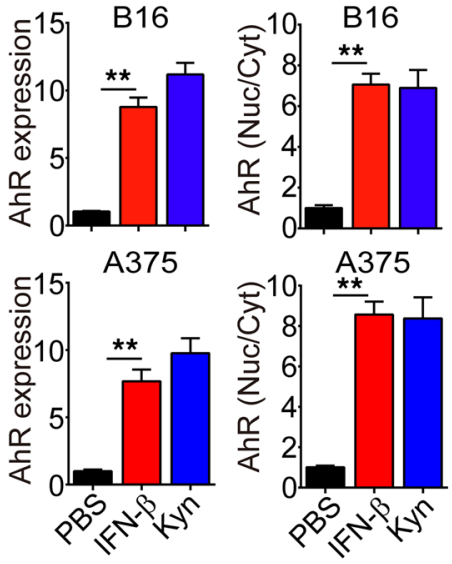
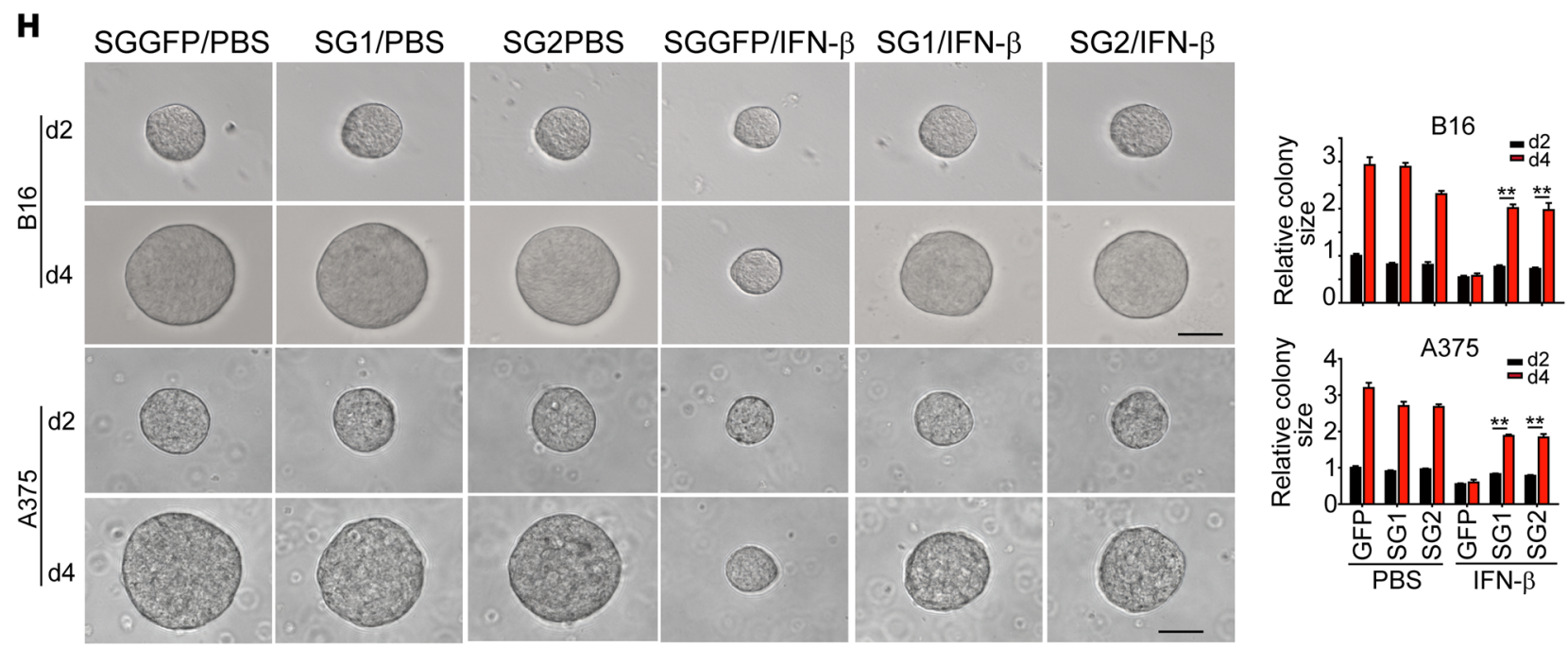

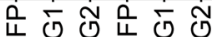

$\frac{0 \text { क क }}{\text { PBS }} \frac{0 \text { क क }}{\text { IFN- } \beta}$ 
Figure 3. IFN- $\beta$ mobilizes ID01/AhR/p27 pathway to mediate TRC dormancy. (A) B16 TRCs or A375 TRCs were treated with IFN- $\beta$ for 24 hours. ID01 expression was determined by Western blot. (B) B16 or A375 cells, cultured on rigid plastic or in soft 3D fibrin gels, were treated with $5 \mathrm{ng} /$ $\mathrm{ml}$ IFN- $\beta$ for 2 days. Kyn levels in cell lysate were determined. (C) IFN- $\beta$ enhanced luciferase activity. B16 TRCs or A375 TRCs were transiently transfected with a luciferase reporter plasmid of $\mathrm{pGL3/CYP1A1} \mathrm{promoter} \mathrm{with}$ or without Flag-AhR in the presence or absence of IFN- $\beta$ for 24 hours. (D) Both IFN- $\beta$ and Kyn promoted the translocation of AhR from the cytosol to the nucleus by immunostaining assay. Scale bar: $10 \mu \mathrm{m}$. (E) Cell fraction of cytosol (Cyt) and nucleus (Nuc) was analyzed by Western blot. (F) Expression of p27 in TRCs or differentiated control (Con) cells with or without IFN- $\beta$ treatment. (G) B16 or A375 TRCs were transfected with a luciferase

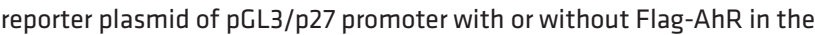
presence or absence of IFN- $\beta$ for 24 hours. (H) Single guide GFP (SGGFP) or p27-sgRNA-TRCs were treated with IFN- $\beta(5 \mathrm{ng} / \mathrm{ml})$ for 2 to 4 days. The colony size is presented. SG1, p27-sgRNA-SG1; SG2, p27-sgRNA-SG2. Scale bars: $50 \mu \mathrm{m}$. Data are from 3 independent experiments and represent mean \pm SEM. ${ }^{* *} P<0.01$, by 2 -tailed Student's $t$ test $(\mathbf{B}, \mathbf{C}$, and $\mathbf{H})$ and 1-way ANOVA (D and $\mathbf{G}$ ).

ylation. In addition to cytokines (IL-6 and IL-10) that induce p-STAT3 (Y), intracellular tyrosine kinases, such as Src kinase, are another important pathway for producing p-STAT3 (Y) (43, 44). Notably, we found that TRCs had a high level of p-Src kinase, which could be brought down by either IFN- $\beta$, Kyn, or the forced overexpression of IDO1 (Figure 5D), suggesting that AhR activity negatively regulates Src kinase phosphorylation. Consistently, inhibition of AhR activity by DMF recovered Src kinase phosphorylation (Figure 5D). Since activated AhR enters the nucleus, where it functions as a transcription factor, we thus further performed ChIP assay to determine the possible transcription regulation of Src kinase by AhR. Indeed, a binding of AhR to the promoter of the Src kinase gene was found (Figure $5 \mathrm{E}$ ). We further confirmed that such binding resulted in the suppression of Src kinase expression, as evidenced by the decreased mRNA level of Src kinase and its rescue by $A h R$ knockout (Supplemental Figure 6D). Moreover, IFN- $\beta$ treatment also inhibited the phosphorylation of Src kinase, which could be reversed by the addition of 1-MT or DMF (Figure $5 \mathrm{~F}$ ). To further verify this, we treated B16 TRCs with IFN- $\beta$ / 1-MT for 48 hours. The immunoprecipitation assay showed that IFN- $\beta / 1$-MT treatment led to Src binding to STAT3 as well as tyrosine-phosphorylated STAT3 (Supplemental Figure 6E). To clarify whether IDO/AhR directly interacts with Src so as to inhibit Src phosphorylation, we treated B16 TRCs with PBS or IFN- $\beta$ for 48 hours, and the immunoprecipitation assay showed that neither IDO1 nor AhR bound to Src (Supplemental Figure 6F). Together, these results suggest that IFN- $\beta$ uses the IDO1/Kyn/AhR pathway to downregulate Src kinase activity so as to decrease STAT3 tyrosine phosphorylation.

Although nuclear translocation of STAT3 is required for its function exertion (45), we found that tyrosine phosphorylation did not mediate the entry of STAT3 into the nucleus in IFN- $\beta$-treated or untreated TRCs (Figure 5G), consistent with the previous report showing that tyrosine phosphorylation is not required for STAT3 nuclear translocation (46). Surprisingly, AhR blockade could induce the entry of p-STAT3 (Y) into the nucleus in IFN- $\beta$-treated TRCs (Figure $5 G$ ). Such paradoxical results promoted us to further investigate the underlying molecular basis. Besides tyrosine phosphorylation, STAT3 also has a serine site for phosphorylation. Intriguingly, IFN- $\beta$ signaling resulted in an increase of serine (S) phosphorylation of STAT3 in TRCs (Figure 5, H and I). Moreover, we found that IFN- $\beta$-promoted STAT3 nuclear translocation was actually mediated by serine phosphorylation: (a) the immunostaining showed that p-STAT3 (S) was localized in the nucleus of IFN- $\beta$-treated TRCs (Figure 5I); (b) Western blot analysis of the cytosolic and nuclear contents showed that p-STAT3 (S) was rare in untreated TRCs, but markedly increased upon IFN- $\beta$ treatment and entered the nucleus (Figure 5J); and (c) more convincingly, serine mutation blocked STAT3 entering the nucleus and tyrosine mutation had no effect (Figure 5K). In addition, since AhR blockade could recover tyrosine phosphorylation, treatment with IFN- $\beta$ plus DMF or 1-MT was expected to generate the dual phosphorylation of tyrosine and serine of STAT3 (Figure 5L). In addition, we treated B16 TRCs with IFN- $\beta(5 \mathrm{ng} / \mathrm{ml})$ or IFN- $\gamma(100 \mathrm{ng} / \mathrm{ml})$ for 48 hours. Cell lysates were extracted for Western blot analysis of STAT1, STAT3, Src, and their phosphorylated forms. The results showed that IFN- $\beta$ decreased p-STAT3 (Y) and p-Src, but increased p-STAT3 (S), p-STAT1, and STAT1, while IFN- $\gamma$, although increasing p-STAT1, had no effect on p-STAT3 (Y), p-STAT3 (S), and p-Src (Supplemental Figure 6G), indicating a complex communication between STAT 3 and STAT1 (IFN- $\beta /$ IFN- $\gamma$ pathways) in TRC dormancy. Together, these data suggested that serine phosphorylation is required for the nuclear translocation of STAT3 to mediate IFN- $\beta /$ AhR inhibitor-induced TRC apoptosis.

p-STAT3 (Y, S) promotes TRC apoptosis through a p53-dependent pathway. Next, we investigated how STAT3 in the nucleus mediated the apoptosis of IFN- $\beta$-treated TRCs by AhR blockade. p53 is a key tumor suppressor guardian that induces tumor cell apoptosis $(47,48)$. Notably, STAT3 has been reported to regulate the expression of p53 $(49,50)$, causing us to speculate that a STAT3/p53 pathway mediates the above-mentioned TRC apoptosis. First, we tried to understand the difference between p-STAT3 (S) induced by IFN- $\beta$ and $\mathrm{p}$-STAT3 (S,Y) expressed following treatment with the IFN- $\beta$ /AhR inhibitor, since both of them could enter the nucleus. By ChIP assay, we found that p-STAT3 (S) and p-STAT3 (S, Y) actually had distinctive roles. p-STAT3 (S, Y) preferentially bound the $\mathrm{p} 53$ promoter and $\mathrm{p}$-STAT3 (S) selectively bound the $\mathrm{p} 27$ promoter (Figure 6A). Intriguingly, p-STAT3 (S) showed its ability to promote $\mathrm{p} 27$, but not $\mathrm{p} 53$, expression in IFN- $\beta$-treated TRCs (Supplemental Figure 7A). On the other hand, p-STAT3 (S, Y) markedly upregulated $\mathrm{p} 53$ expression, but not $\mathrm{p} 27$, in IFN- $\beta / \mathrm{AhR}$ inhibitortreated TRCs (Supplemental Figure 7A). Notably, we found that only $p 53$ knockdown, but not $p 27$ knockout, saved IFN- $\beta$-treated TRCs from apoptosis induced by DMF or 1-MT (Figure 6B), suggesting that p-STAT3 $(\mathrm{S}, \mathrm{Y})$ in the nucleus triggers TRC apoptosis through a p53-dependent pathway. To further confirm this, we knocked out STAT3 in B16 TRCs and then stably transduced Flag-WT-STAT3, Flag-Y705F-STAT3 (tyrosine mutation), or Flag-S727A-STAT3 (serine mutation) into the cells. The ChIPquantitative PCR (ChIP-qPCR) assay showed that, upon IFN- $\beta$ treatment, both Flag-WT-STAT3 and Flag-Y705F-STAT3 bound to the promoter of p27, while Flag-S727A-STAT3 had no specific binding to the promoter of p27 (Figure 6C and Supplemental Figure $7 \mathrm{~B}$ ), indicating that serine phosphorylation indeed mediates the binding of STAT3 to the p27 promoter; upon IFN- $\beta / 1$-MT 

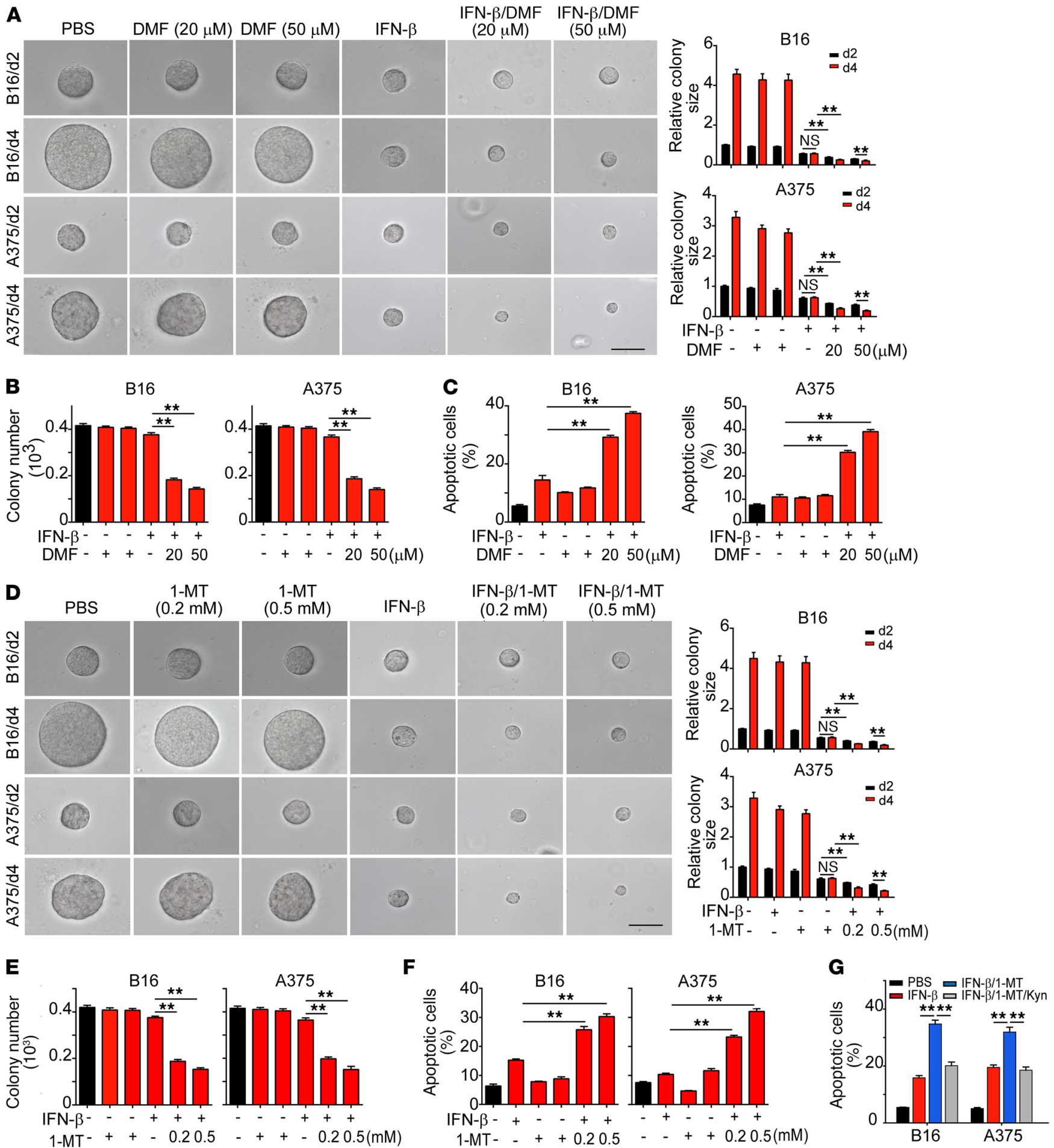

Figure 4. Blockade of ID01/AhR pathway abrogates IFN- $\beta$-induced dormant TRCs. (A and B) B16 or A375 TRCs were seeded in soft 3D fibrin gels. Two days later, IFN- $\beta$ was added for a further 2 days or 4 days of culture in the presence or absence of DMF. The colony size (A) was presented relative to the PBS (day 2) group, which was set to 1 , and colony number (B) was counted. Scale bars: $50 \mu \mathrm{m}$. (C) B16 or A375 TRCs were treated with IFN- $\beta$ (5 ng/ml) with or without DMF for 48 hours. Cell apoptosis was determined by flow cytometry. (D and E) B16 or A375 TRCs were treated with PBS, IFN- $\beta, 1-M T$, or IFN- $\beta$ + 1-MT for indicated times. The colony size was measured (D), and colony number was counted (E). Scale bar: $50 \mu \mathrm{m}$ (D). (F and G) B16 or A375 TRCs were treated with IFN- $\beta$ ( $5 \mathrm{ng} / \mathrm{ml}), 1-\mathrm{MT}$, IFN- $\beta+1-\mathrm{MT}$, or IFN- $\beta+1-\mathrm{MT}+\mathrm{Kyn}(200 \mu \mathrm{M})$ for 48 hours. Cell apoptosis was determined by flow cytometry. Data are from 3 independent experiments. Graphs represent mean \pm SEM. ${ }^{* *} P<0.01$, 1-way ANOVA (A-G). 
A

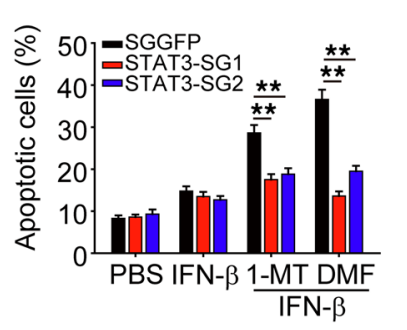

D

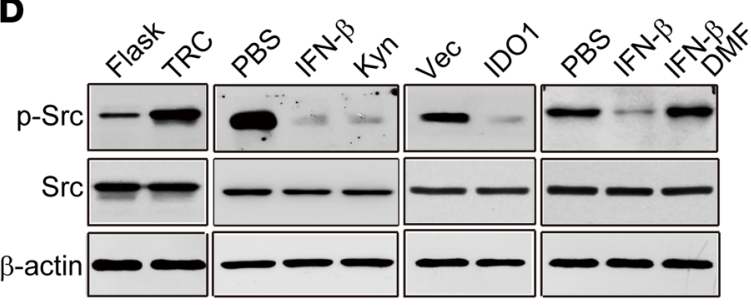

G
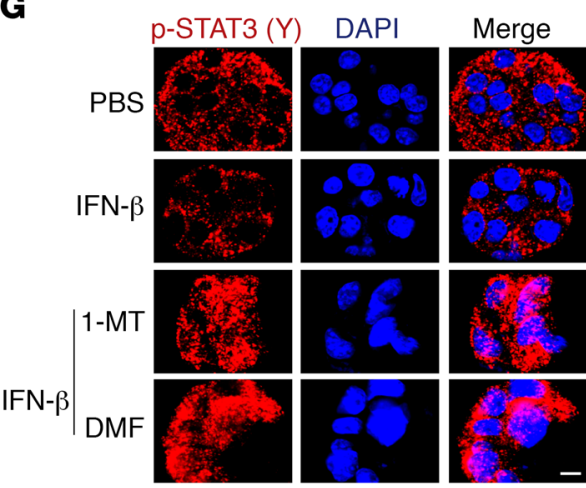

J
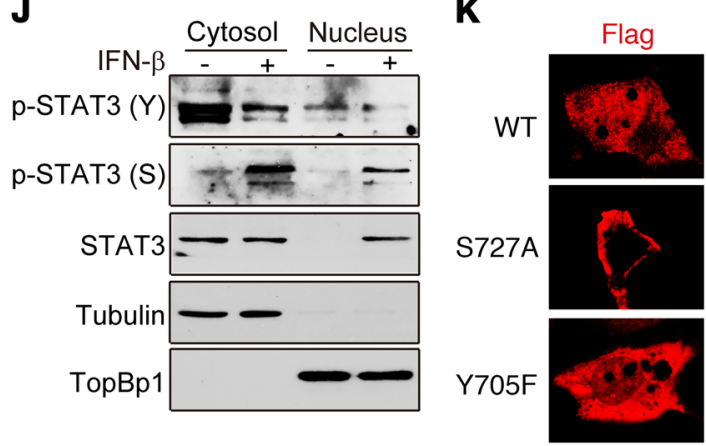

B

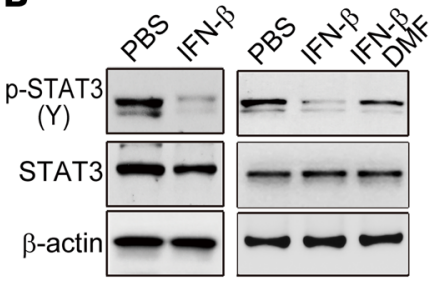

C

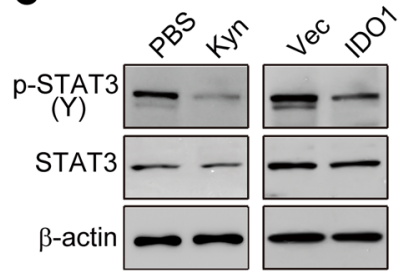

E

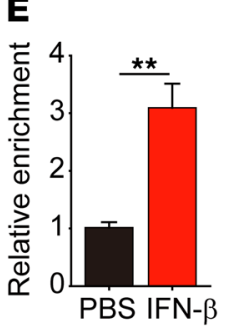

$\mathbf{F}$

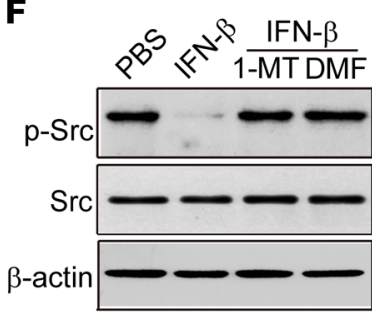

H

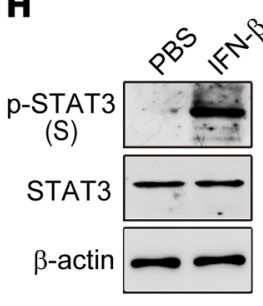

I

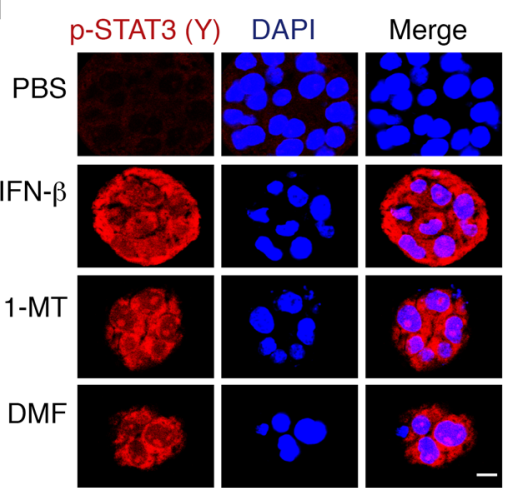

$\mathbf{L}$
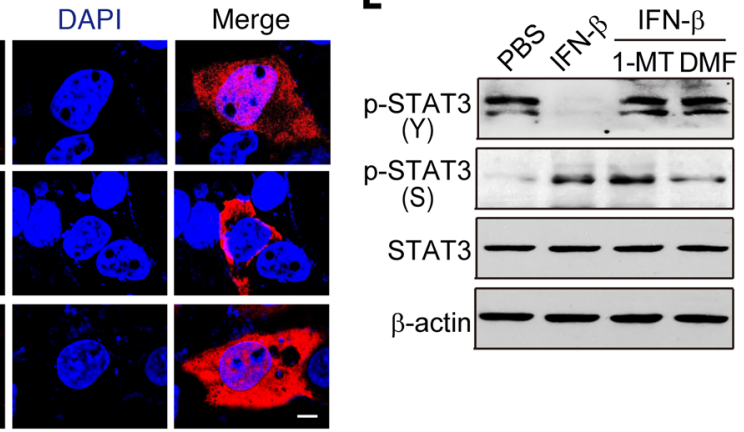

Figure 5. Nuclear translocation of p-STAT3 (S) mediates TRC apoptosis by IFN- $\beta$ /AhR blockade. (A) B16 TRCs with or without STAT3 knockout were treated with PBS, IFN- $\beta$, IFN- $\beta / 1-M T$, or IFN- $\beta / D M F$. Apoptosis was analyzed by flow cytometry. (B) p-STAT3 (Y) and STAT3 were determined by Western blot in B16 TRCs treated with IFN- $\beta$ or IFN- $\beta$ /DMF. (C) Western blot analysis of p-STAT3 (Y) and STAT3 in B16 TRCs treated with PBS or Kyn, Vec-B16 TRCs, or ID01-B16 TRCs. (D) Western blot analysis of p-Src and Src from B16 cells, B16 TRCs, ID01-overexpressing B16 TRCs, B16 TRCs treated with PBS, IFN- $\beta$, Kyn, or IFN- $\beta$ / DMF. (E) ChIP-qPCR analysis was performed with anti-AhR and primers specific for Src in IFN- $\beta$-pretreated B16 TRCs. (F) Western blot analysis of p-Src and Src in B16 TRCs treated with PBS, IFN- $\beta$, IFN- $\beta / 1-M T$, or IFN- $\beta /$ DMF. (G) $p$-STAT3 (Y) and DAPI immunostaining in IFN- $\beta$-treated B16 TRCs. Scale bar: $10 \mu$ m. (H) Western blot analysis of p-STAT3 (S) and STAT3 in B16 TRCs treated with IFN- $\beta$. (I) As in G, B16 TRCs were immunostained against p-STAT3 (S) and DAPI. Scale bar: $10 \mu \mathrm{m}$. (J) B16 TRCs were treated with IFN- $\beta$ or PBS. Cytosolic and nuclear p-STAT3 (Y), p-STAT3 (S), and STAT3 were analyzed. (K) B16 TRCs were transfected with Flag-WT-STAT3, Flag-Y705F-STAT3, or Flag-S727A-STAT3 and then treated with IFN- $\beta$ for 24 hours. Flag and DAPI were visualized by immunostaining. Scale bar: $5 \mu \mathrm{m}$. (L) Same conditions as in F. The expression of p-STAT3 (Y), p-STAT3 (S), and STAT3 were analyzed by Western blot. Graphs represent mean \pm SEM of 3 assays. ${ }^{*} P<0.01$, by 1-way ANOVA $(\mathbf{A})$ and 2 -tailed Student's $t$ test (E).

treatment, only Flag-WT-STAT3 bound to the p53 promoter and neither tyrosine- nor serine-mutated STAT3 was found to bind to the $\mathrm{p} 53$ promoter (Figure 6C), indicating that dual phosphorylation of serine and tyrosine is required for STAT3 to bind to the p53 promoter. Meanwhile, Western blot analysis showed that tyrosine-mutated STAT3, but not serine-mutated or WT-STAT3 TRCs, expressed the highest p27 upon IFN- $\beta$ treatment, while treatment with IFN- $\beta / 1$-MT or IFN- $\beta /$ DMF was found to upreg- 
A
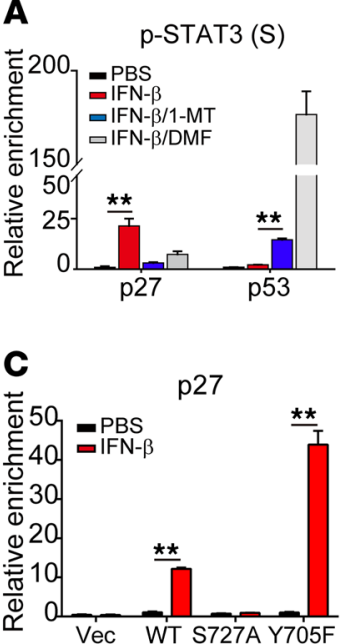
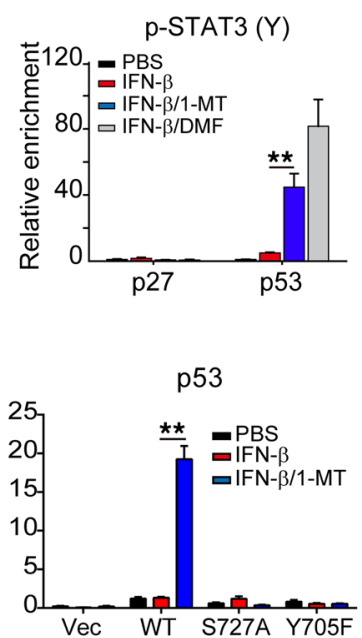

B

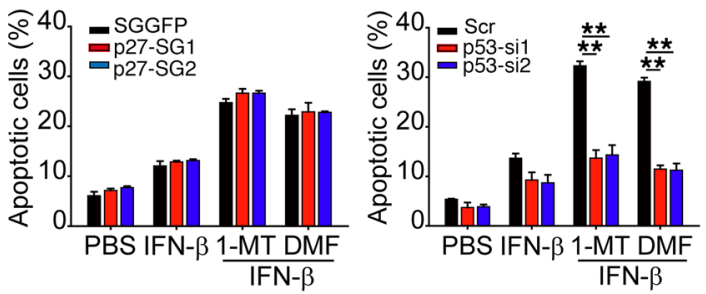

E

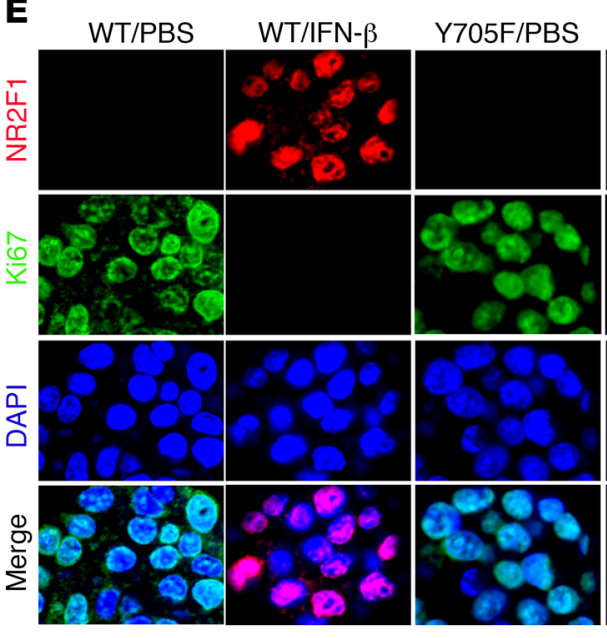

D

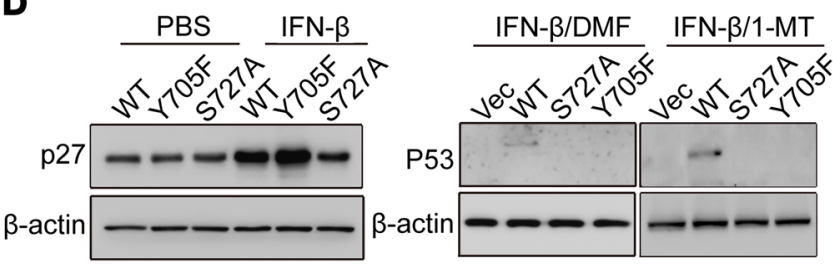

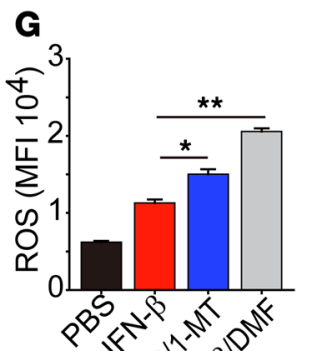

H

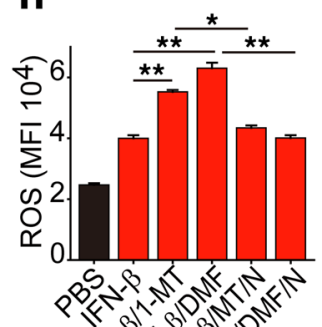

से

J
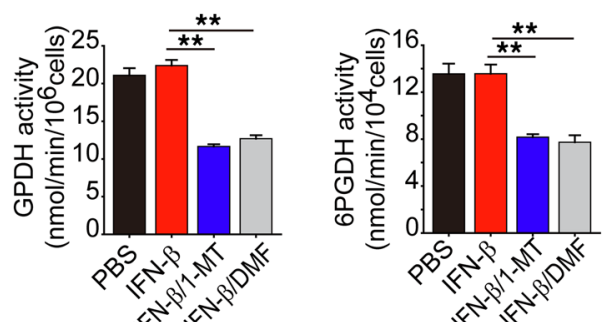

rescrstrus

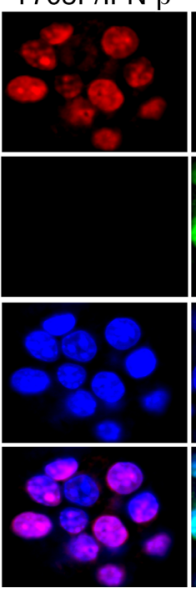

I
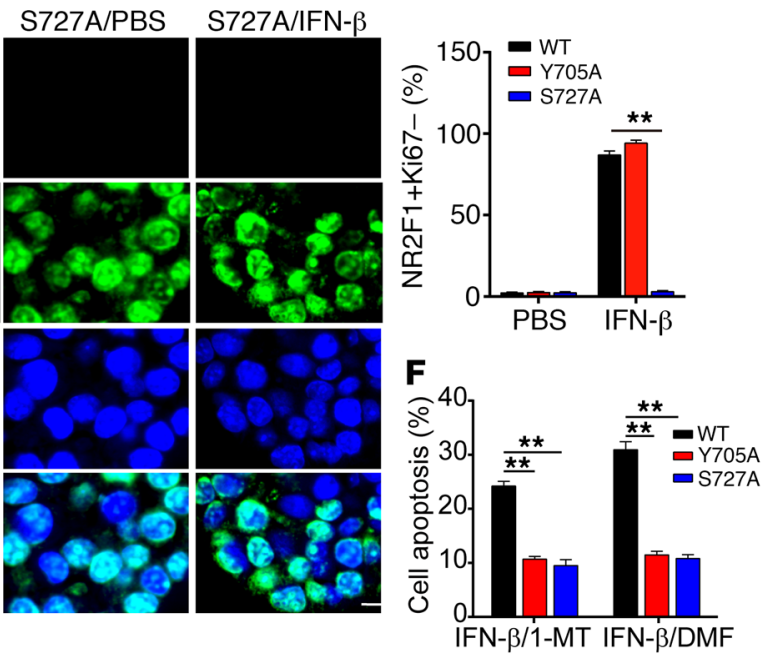

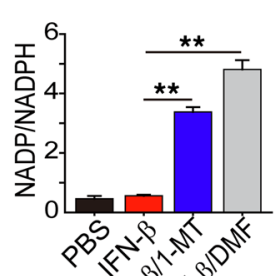

साइ

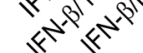

\section{K}
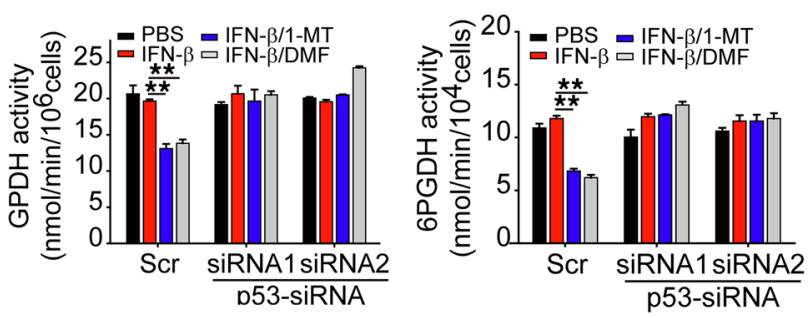
Figure 6. STAT3/p53 pathway regulates IFN- $\beta$-induced TRC apoptosis. (A) B16 TRCs were treated with PBS, IFN- $\beta$, IFN- $\beta / 1-M T$, or IFN- $\beta / D M F$ for 48 hours. Cells were collected for ChIP-qPCR assay with anti-p-STAT3 (S) (left) or p-STAT3 (Y) (right) and specific primer of p27 or p53. (B) B16-p27SGGFP and B16-p27-SGs TRCs (left) or scramble-B16 and p53-siRNAs-B16 TRCs (right) were treated with IFN- $\beta$, IFN- $\beta / 1-M T$, or IFN- $\beta$ /DMF for 24 hours. Cell apoptosis was determined by flow cytometry. (C) STAT3-sgRNA-B16 cells stably expressing Flag-WT-STAT3, Flag-Y705F-STAT3, or FlagS727A-STAT3 were seeded in 3D fibrin gels and then treated with IFN- $\beta$ or IFN- $\beta / 1-M T$. After 48 hours of treatment, Chip-qPCR assay was performed by using anti-Flag antibody with specific primer for p27 or p53. (D and E) B16 TRCs stably overexpressing Flag-WT-STAT3, Flag-Y705F-STAT3, or Flag-S727A-STAT3 were treated with PBS, IFN- $\beta$, IFN- $\beta / 1-M T$, or IFN- $\beta$ / DMF for 48 or 72 hours. Cell lysates (48 hours) were extracted for Western blot (D) or (72 hours) were fixed and immunostained with anti-NR2F1 and Ki67 antibodies (E) Scale bar: $10 \mu \mathrm{m}$. (F) As in (D), but cell apoptosis was determined by flow cytometry. (G-I) B16 TRCs were treated with PBS, IFN- $\beta$, IFN- $\beta / 1-M T$, IFN- $\beta / D M F$, IFN- $\beta / 1-M T / N A C$ ( $10 \mathrm{mM}$ ), or IFN- $\beta / D M F /$ NAC for 48 hours. ROS levels were determined by flow cytometry (G and H). Levels of GSH, GSSG, NADP, or NADPH were measured and the ratio of GSSG/GSH or NADP/NADPH was calculated (I). (J) Activity of GPDH and $6 \mathrm{PGDH}$ in B16 TRCs treated with PBS, IFN- $\beta$, IFN- $\beta / 1-\mathrm{MT}$, or IFN- $\beta / D M F$ for 48 hours. (K) B16 TRCs were transfected with p53-siRNA for 24 hours and then treated with IFN- $\beta$, IFN- $\beta / 1-M T$, or IFN- $\beta / D M F$ for 48 hours. The activity of GPDH and 6PGDH was measured. Graphs represent mean \pm SEM of 3 independent experiments. ${ }^{*} P<0.05$; ${ }^{* *} P<0.01$, by 1 -way ANOVA (A-C and E-K).

ulate p53 expression in TRCs transduced with WT-STAT3, but not with tyrosine- or serine-mutated STAT3 (Figure 6D). In addition, upon low-dose $(2.5 \mathrm{ng} / \mathrm{ml})$ IFN- $\beta$ treatment, WT- and Y705A-STAT3-B16 TRCs, but not S727A-STAT3-B16 TRCs, were induced into dormancy, as evaluated by the NR2F1+Ki67 dormancy marker (Figure 6E). Meanwhile, we found that tyrosine or serine mutation inhibited TRC apoptosis induced by IFN- $\beta$ /1-MT or DMF (Figure 6F). Here, we also clarified the regulatory roles of STAT3 and IDO-AhR in p27 expression. We treated STAT3-knockout B16 TRCs with low or high concentrations of IFN- $\beta$ ( 2.5 or $10 \mathrm{ng} / \mathrm{ml}$ ) for 48 hours. Western blot analysis of cell lysates showed that, in the control TRCs, p27 expression was markedly upregulated by both 2.5 and $10 \mathrm{ng} / \mathrm{ml}$ IFN- $\beta$, while in STAT3-knockout TRCs, although 2.5 ng IFN- $\beta$ slightly upregulated p27 expression, 10 ng IFN- $\beta$ markedly upregulated p27 expression (Supplemental Figure 7C). Then, we further treated STAT3-knockout B16 TRCs with IFN- $\beta$ in the presence of 1-MT or DMF for 48 hours. We found that blockade of IDO1 or AhR abrogated the effect of IFN- $\beta$ on p27 upregulation (Supplemental Figure 7D). These results suggest that the IDO/AhR pathway is indeed involved in IFN- $\beta$-mediated p27 upregulation as well as TRC dormancy.

ROS is an important pathway that mediates tumor cell apoptosis. Given the regulatory effect of p53 on ROS production (51, 52 ), we focused on ROS levels in TRCs. Intriguingly, we found that AhR blockade significantly increased ROS levels in IFN- $\beta$-treated TRCs (Figure 6G and Supplemental Figure 7E). The use of antioxidant agent $N$-acetyl cysteine (NAC) not only decreased ROS levels, but also blocked the apoptosis of IFN- $\beta$-treated TRCs induced by DMF or 1-MT (Figure 6H and Supplemental Figure 7, E and F), leading to colony regrowth and an increase in colony number (Supplemental Figure $7 G$ ). The glutathione (GSH) system is the most important antioxidative system. The thiol group of GSH can react with ROS and is then oxidized into GSH disulfide (GSSG). In line with the increase of ROS, AhR blockade was found to influence the GSH system and an increased ratio of GSSG/GSH was seen in IFN- $\beta /$ AhR inhibitor-treated TRCs, concomitant with decreased levels of NADPH (Figure 6I), an important molecule required to reduce GSSG to GSH. NADPH is mainly produced through the pentose phosphate pathway (PPP) by glucose-6-phosphate dehydrogenase $(\mathrm{GPDH})$, which catalyzes the first step of PPP and 6-phosphogluconate dehydrogenase (6PGDH). Intriguingly, IFN- $\beta$ alone did not affect the expression of these 2 enzymes; however, IFN- $\beta$ plus DMF/1-MT significantly downregulated both GPDH and 6PGDH mRNA expression and activity (Figure 6J and Supplemental Figure $7 \mathrm{H}$ ), consistent with a previous report showing that $\mathrm{p} 53$ prevents the formation of the active dimer of GPDH (53). Moreover, such downregulation of GPDH and 6PGDH was reverted by $p 53$ knockdown (Figure $6 \mathrm{~K}$ and Supplemental Figure 7I). Regarding the possible influence of mutation on p53-regulated PPP, we used mutant $\mathrm{p} 53$ cell lines, including MDA-MB-231 (breast cancer, p53R280K) and SGC-7901 (stomach cancer, p53E204A) cancer cells. Consistently, treatment of these p53-mutated TRCs with IFN- $\beta / 1-$ MT or IFN- $\beta / D M F$ remarkedly inhibited the colony size and decreased the colony number (Supplemental Figure 8, A and B). Also, IFN- $\beta / 1-\mathrm{MT}$ or IFN- $\beta / \mathrm{DMF}$ treatment remarkedly increased ROS levels and GSSG/GSH and NADP/NADPH ratios as well as decreased the activity of GPDH and 6PDGH in these p53-mutated cells (Supplemental Figure 8, C-G). Thus, although p53 mutation is frequent in tumor cells, it seems not to influence p53 regulation of PPP in TRCs. Together, these data suggest that p-STAT3 (S, Y) in the nucleus binds to the p53 promoter, inducing the expression of p53, and that the latter interferes with the cellular redox system by downregulating GPDH, leading to disruption of IFN- $\beta$-induced TRC dormancy.

Combining IFN- $\beta$ and an AhR inhibitor disrupts melanoma TRC dormancy in vivo. The experiments discussed above showed that blockade of AhR activity led to switching IFN- $\beta$-induced TRC dormancy into disruption through a STAT3/p53-dependent pathway. Here, we further verified this process in vivo. Previous data showed that intratumoral injection of IFN- $\beta$ could enrich TRCs and induced most of them into dormancy (Figure $1, A-G$ ). Here, we further confirmed that intratumoral injection of IFN- $\beta$ resulted in the upregulation of IDO1, AhR, p27, and p-STAT3 (S) expression and the downregulation of p-STAT3 (Y) expression in B16 (Figure 7A) or A375 (Supplemental Figure 9A) melanoma-bearing mice and translocation of AhR and p-STAT3 (S) into the nucleus of B16 cells (Figure 7B) or A375 cells (Supplemental Figure 9B), suggesting that IFN- $\beta$ uses the IDO1/AhR/p27 pathway to induce melanoma TRC dormancy in vivo. In support of this notion, immunocostaining by anti-AhR and anti-CD133 also showed the nuclear translocation of $\mathrm{AhR}$ in $\mathrm{CD} 133^{+} \mathrm{B} 16$ or A375 tumor cells upon IFN- $\beta$ treatment (Supplemental Figure 9C). Next, we looked into whether those IFN- $\beta$-induced in vivo dormant TRCs could be abrogated through blockade of the IDO/AhR pathway. C57BL/6 mice were injected s.c. with $1 \times 10^{4} \mathrm{~B} 16$ melanoma TRCs, and once tumors reached a size of $7 \times 7 \mathrm{~mm}$, animals were treated with IFN- $\beta$ and an IDO1 (1-MT) or an AhR inhibitor (DMF) for 8 days. We then isolated CD133 ${ }^{\text {hi }}$ tumor cells from the tumor mass and 
A
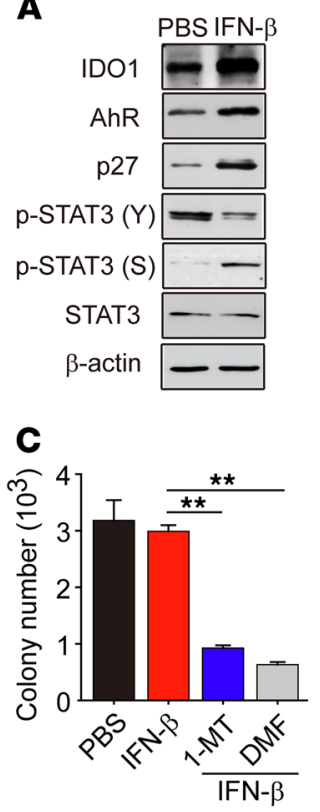

D

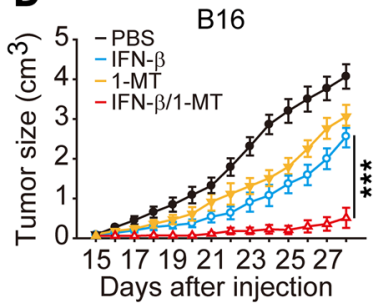

$\mathbf{F}$

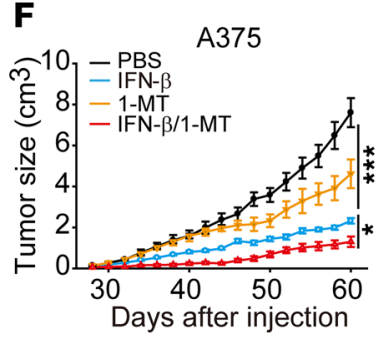

B
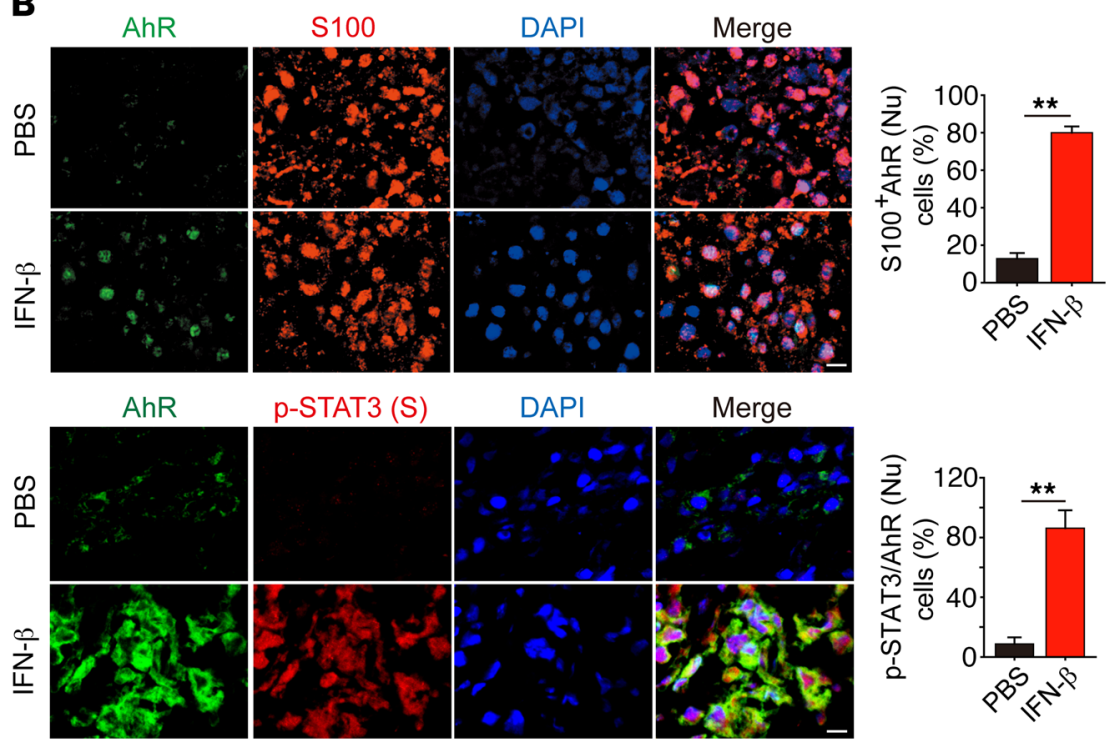

p-STAT3 (S)

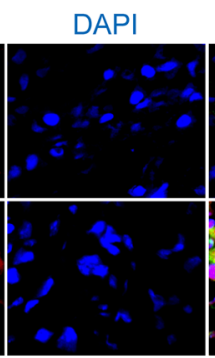

Merge
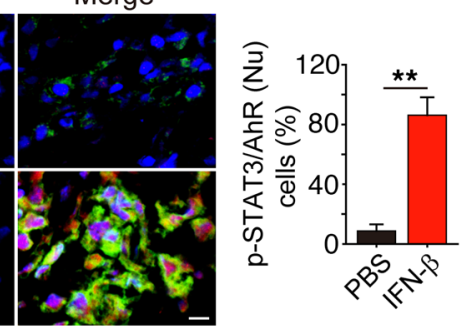

Figure 7. IFN- $\beta$ /AhR blockade improves survival in vivo. (A) Mice with $5 \times 5$ mm B16 melanoma were treated with IFN- $\beta$ daily for 3 days. ID01, AhR, p27, p-STAT3 (Y), p-STAT3 (S), and STAT3 were analyzed by Western blot in tumor cells $(n=5)$. (B) As in A, but tumor tissue was immunostained with antiAhR, S100, and p-STAT3 (S), and DAPI. The percentage of cells with AhR nuclear localization was determined among 500 S100 cells in 10 nonconsecutive sections (upper right panel). The number of cells with both AhR and p-STAT3 (S) nuclear localization was counted (lower right panel) ( $n=5$ ). Scale bars: 10 $\mu \mathrm{m}$. (C) Mice with $7 \times 7 \mathrm{~mm}$ B16 melanoma were treated with IFN- $\beta$, IFN- $\beta / 1-M T$, or IFN- $\beta / D M F$ for 8 days. CD133 ${ }^{\text {hi }}$ tumor cells isolated were seeded in soft $3 D$ fibrin gels. Colony number was counted on day $4(n=5)$. ( $\mathbf{D}$ and $\mathbf{E})$ Mice were inoculated with $1 \times 10^{5}$ unsorted B16 cells. When tumor size was $7 \times 7$ mm, mice were treated with IFN- $\beta, 1-M T$, or IFN- $\beta / 1-M T$ (D) or IFN- $\beta$, DMF, or IFN- $\beta / D M F(E)$ for 10 days. Tumor growth was measured and long-term survival was analyzed $(n=10)$. (F and G) NOD-SCID mice with $7 \times 7$ mm A375 melanoma were treated with IFN- $\beta, 1-M T$, or IFN- $\beta / 1-M T(F)(n=8)$ or IFN- $\beta$, DMF, or IFN- $\beta$ /DMF (G) for 10 days $(n=8)$. Data represent mean \pm SEM. ${ }^{*} P<0.05$; ${ }^{* *} P<0.01 ;{ }^{* *} P<0.001$ and ${ }^{\# \#} P<0.01$ vs. IFN- $\beta$-treated group, by 2 -tailed Student's $t$ test (B), 1-way ANOVA (C-G, left panels of $\mathbf{D}$ and $\mathbf{E})$, and Kaplan-Meier survival analysis (D and E, right panels).

seeded them into soft 3D fibrin gels. The result showed that IFN- $\beta$ plus 1-MT or DMF treatment significantly reduced the number of colonies formed, compared with IFN- $\beta$ treatment alone (Figure $7 C$ ). In addition, the isolated $C D 133^{\text {hi }} \mathrm{B} 16$ cells showed much lower GPDH and 6PGDH enzymatic activity, but much higher ROS levels in the IFN- $\beta / 1-M T$ or DMF treatment group (Supplemental Figure 9D), suggesting that TRCs are abrogated by the combined treatment. In line with this, the combined treatment (IFN- $\beta$ plus 1-MT or DMF) was found to be superior to each single treatment by significantly repressing tumor growth and prolong- ing the survival of mice (Figure 7, D and E). Furthermore, we also validated these results with human melanoma by s.c. injection of A375 TRCs into NOD-SCID mice. Similarly, the combining therapy of IFN- $\beta$ and 1-MT or DMF remarkably inhibited A375 melanoma (Figure 7, $\mathrm{F}$ and $\mathrm{G}$ ), demonstrating that IFN- $\beta$ and the IDO1/ AhR inhibitor have remarkable treatment efficiency in melanoma. In addition to melanoma, large $\mathrm{H} 22$ hepatocellular carcinoma (7 $\times 7 \mathrm{~mm})($ Supplemental Figure 9, E and F) or MCF7 breast carcinoma $(7 \times 7 \mathrm{~mm})$ (Supplemental Figure 9G) was also effectively treated by the combination of IFN- $\gamma$ and 1-MT or DMF. Here, we 
also treated NOD-SCID mice bearing p53-mutated MDA-MB-231 or SGC-7901 tumor $(5 \times 5 \mathrm{~mm})$ with the above regimens for 10 days. Consistently, IFN- $\beta / 1-\mathrm{MT}$ or IFN- $\beta / \mathrm{DMF}$ significantly retarded tumor growth (Supplemental Figure $9 \mathrm{H}$ ). To clarify the role of IFN- $\beta$-induced TRC dormancy in tumor outcomes following the treatments described above, we treated B16 melanomabearing NOD-SCID mice once per day for 3 days using $250 \mathrm{ng} / \mathrm{ml}$ IFN- $\beta$. Under these immunodeficiency conditions, B16 TRCs were also induced into dormancy, evaluated by $\mathrm{G}_{0} / \mathrm{G}_{1}$ arrest (Supplemental Figure 9I) and $\mathrm{CD} 133^{+} \mathrm{NR} 2 \mathrm{~F}^{+}{ }^{+} \mathrm{Ki} 67^{-}$dormant markers (Supplemental Figure 9J), suggesting that IFN- $\beta$-induced TRC dormancy is independent of antitumor immune response. Together, these data suggest that IFN- $\beta$ induced tumor dormancy in vivo by activating the IDO1/AhR pathway and that blocking this pathway produces an ideal treatment approach against cancers by combining with IFN- $\beta$ treatment.

Blocking IDO1/AhR abrogates dormant primary human melanoma TRCs by IFN- $\beta$. Next, we investigated whether the mechanism underlying IFN- $\beta$-induced TRC dormancy could be translated to human TRCs. To this end, single melanoma cells isolated from a melanoma patient were seeded into soft 3D fibrin gels to grow TRCs, as described in previous reports, and then these TRCs were treated with IFN- $\beta$. Consistently, such human primary melanoma TRCs were also induced into dormancy, as evidenced by their growth inhibition, increases in the percentage of NR2F1 ${ }^{+} \mathrm{Ki} 67^{-}$cells and cell-cycle arrest at the $G_{0} / G_{1}$ phase (Figure $8, A^{-} C$ ), decreases in glucose consumption, and the absence of senescence (Supplemental Figure 10, A and B). Furthermore, upon IFN- $\beta$ treatment, these primary melanoma TRCs also upregulated the expression of IDO1 and AhR and increased the nuclear translocation of AhR (Figure 8D). In line with AhR entering the nucleus, p27 and p-STAT3 (S) expression were markedly upregulated, while p-STAT3 (Y) expression was reduced (Supplemental Figure 10C). Moreover, the primary human melanoma TRCs could be induced into dormancy by Kyn (Supplemental Figure 10D); however, the addition of AhR inhibitor DMF blocked this dormant process (Supplemental Figure 10D), further corroborating that IFN- $\beta$ drives primary human melanoma TRC into dormancy through the IDO1/AhR/p27 pathway. We then combined IFN- $\beta$ and 1-MT or DMF to treat these primary human melanoma TRCs in order to clarify whether the induced dormancy can be abrogated. As shown in Supplemental Figure $10, \mathrm{E}$ and $\mathrm{F}$, the combination of IFN- $\beta$ and $1-\mathrm{MT}$ or DMF significantly decreased colony size and colony number of the TRCs. To further validate these results, NOD-SCID mice were inoculated with $1 \times 10^{5}$ primary human melanoma TRCs, followed by combined IFN- $\beta$ and 1-MT treatment. We found this combined treatment strikingly inhibited tumor growth compared with control groups (Figure $8 \mathrm{E}$ ). Consistent treatment results were also obtained from mice inoculated with bulk primary human melanoma cells (Supplemental Figure 10G). These results indicate that exogenous provision of IFN- $\beta$ is able to induce human TRC dormancy through the IDO1/AhR/ p27 pathway and may generate therapeutic outcomes, especially in combination with the AhR inhibitor. Here, we further explored whether endogenous IFN- $\beta$ also induced TRCs into dormancy in cancer patients. Using a bioinformatic approach, we analyzed IFN- $\beta$ expression in patients with different types of cancers, including colon, stomach, breast, lung, pancreatic, and urothelial cancers, and melanoma. The result showed that some tumor samples indeed expressed IFN- $\beta$ (Figure 8F). To validate this, we conducted immunohistochemical staining of IFN- $\beta$ in clinical melanoma samples $(n=10)$ and found that IFN- $\beta$ was weakly expressed in 6 samples and highly expressed in 4 samples (Figure 8G). Intriguingly, in the samples with IFN- $\beta^{\text {hi }}$ expression, the proportion of $\mathrm{NR} 2 \mathrm{~F}^{+}{ }^{+} \mathrm{Ki} 67^{-}$dormant cells was remarkably elevated; in contrast, in the samples with IFN- $\beta^{\text {lo }}$ expression, the proportion of NR2F1-Ki67 $7^{+}$proliferating cells was strikingly high (Figure $8 \mathrm{H}$ ). In addition, IFN- $\beta$ high expression was found to be correlated with better survival in melanoma patients (Figure 8I). Together, these data suggest that endogenous IFN- $\beta$ can also induce human melanoma TRC dormancy.

\section{Discussion}

Despite the great success of current $\mathrm{T}$ cell- and antibody-based immunotherapies for cancer treatment, mobilizing the innate arm of the immune system against cancers remains a challenge. Type I IFNs are commonly expressed in broad cell types upon viral infection and function as the essential innate immune arm against these pathogens by not only directly interfering with viral replication, but also activating innate immune cells and promoting adaptive immune responses (1). These immuno-modulatory effects are widely credited for type I IFNs' ability to act as an antitumor agent and suppress tumor growth (5-7). Despite the roles in promoting antitumor immune responses, in the present study, we provide evidence that IFN- $\beta$ actually can directly target stem-like TRCs and drive them into dormancy through an AhR-mediated pathway and that blocking AhR leads to the disruption of IFN- $\beta$-induced TRC dormancy (Figure 8J). These findings uncover a critical, previously unknown antitumor role of IFN- $\beta$, thus opening the door to a new tumor immunotherapeutic strategy with potential clinical applications.

Entry into dormancy might be an important biological event through which cells are able to survive in extremely harsh environments (31). Transformed malignant cells, regardless of their many acquired cancer hallmarks such as genomic instability, altered metabolism, unregulated proliferation, and antiapoptotic capacity, actually not only suffer the rejection mediated by local normal biophysical and biochemical environments, but also face attack from the immune system (54). Thus, many tumor cells might enter a state of dormancy for their survival during the natural process of tumor evolution under immune surveillance and in response to antitumor therapy. Currently, tumor dormancy is divided into 2 models: (a) tumor mass dormancy by the balance of tumor cell proliferation and death mediated by poor vascularization, immune cell killing, or chemo-radiotherapy and (b) cellular dormancy by either solitary cells or a small group of cells entering quiescence. Although primary tumor cells entering a dormant state in the distant organ may delay the growth of metastatic tumor, it also hinders the killing by chemotherapeutic drugs or cytotoxic immune cells (12, 32). Thus, elucidation of dormancy mechanisms and elimination of dormant tumor cells would be a very important and promising approach for treating primary tumors and preventing metastatic relapse. Unlike immune cell killing that induces tumor mass dormancy, induction of tumor cell quiescence by immune factors is another form of immunologic dormancy. In this study, we demonstrate that IFN- $\beta$, a pivotal innate cytokine, effectively induces stem-like TRCs into dormancy. Regarding this finding, a key ques- 
A

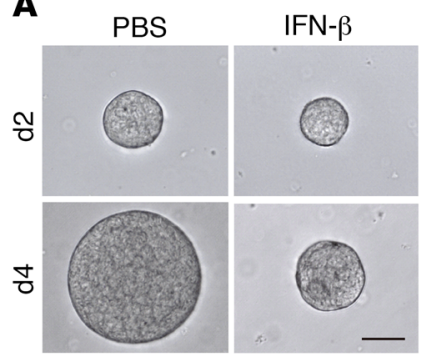

C

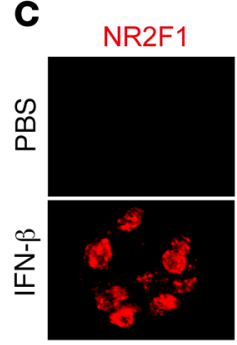

D
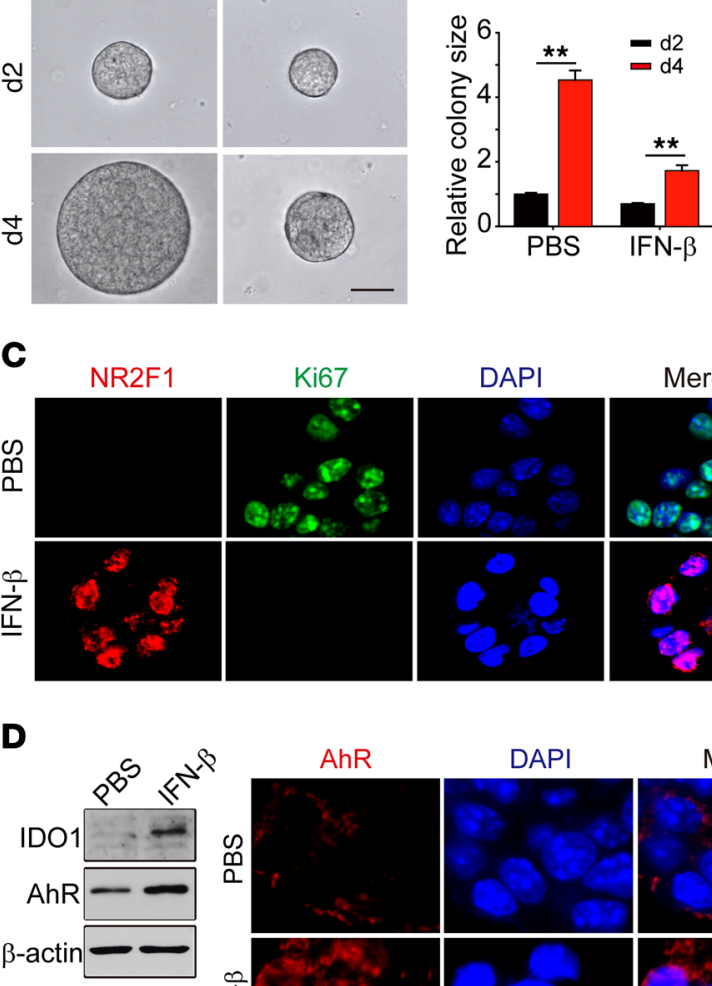

B
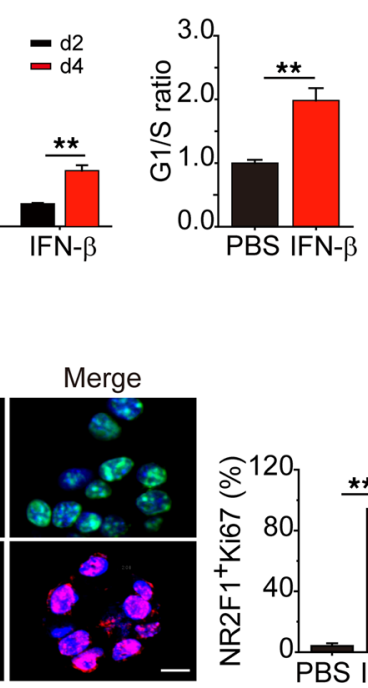

H
G

$\mathrm{P} 1^{\#}$

$\mathrm{P} 2^{\#}$

$\mathrm{P}^{\#}$

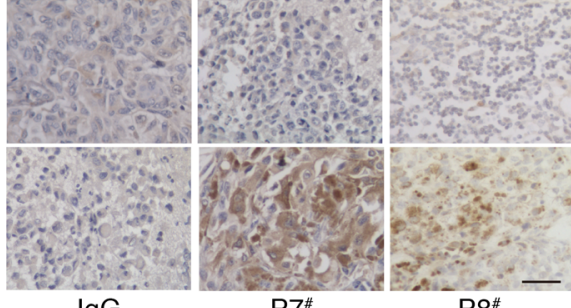

$\lg G$

$\mathrm{P}^{\# \#}$

$\mathrm{P}^{\#}$
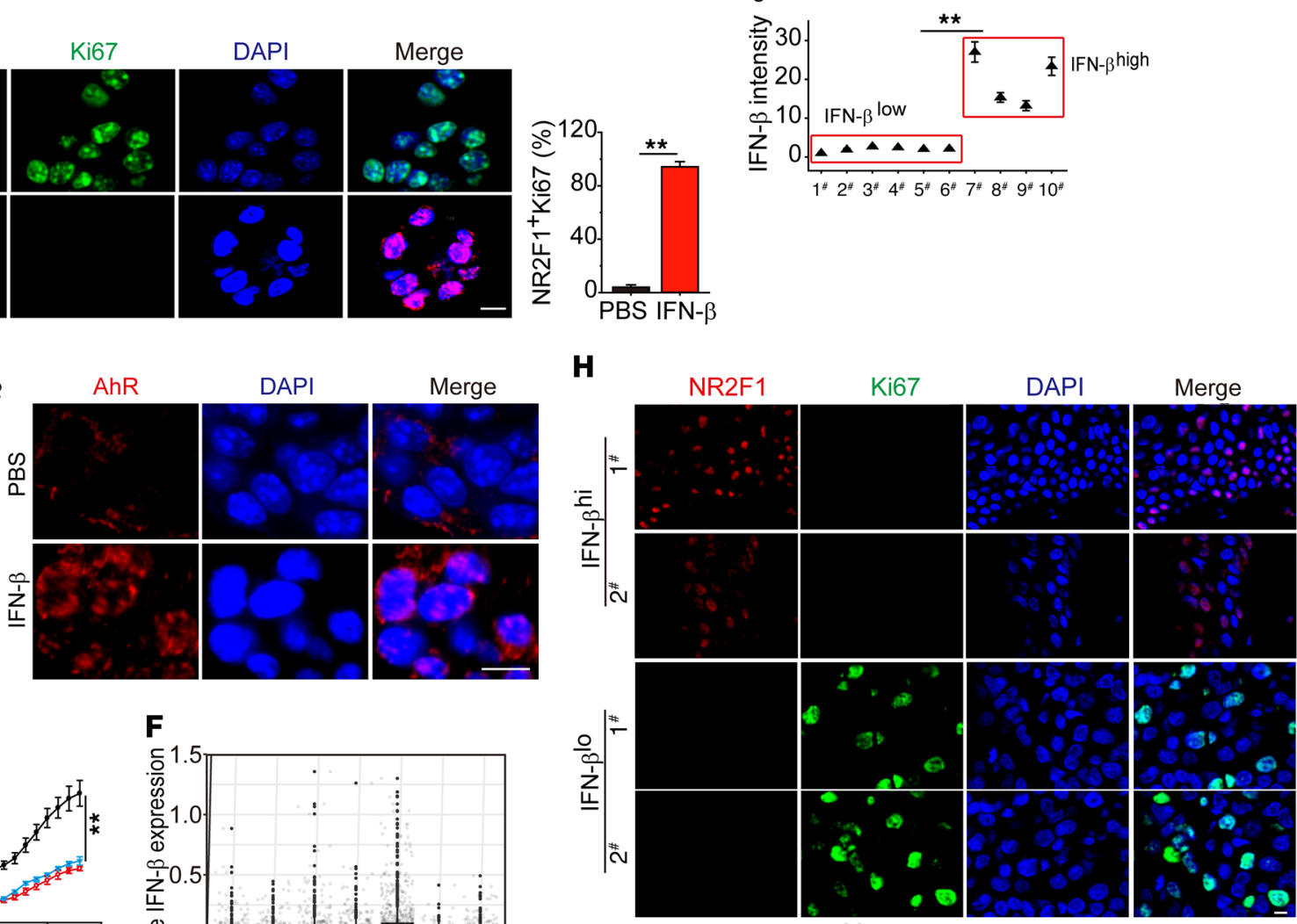

E

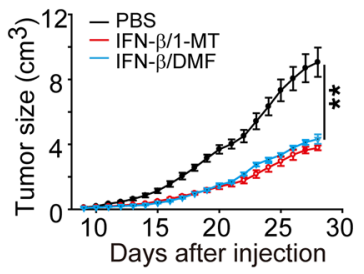

F
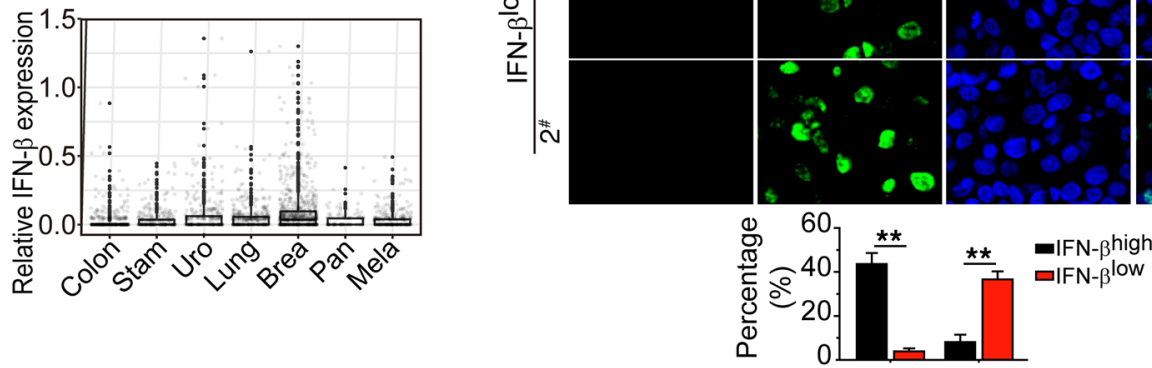

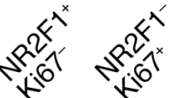

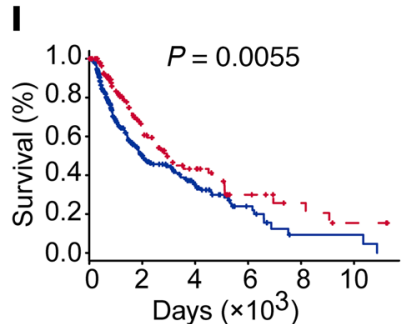

J

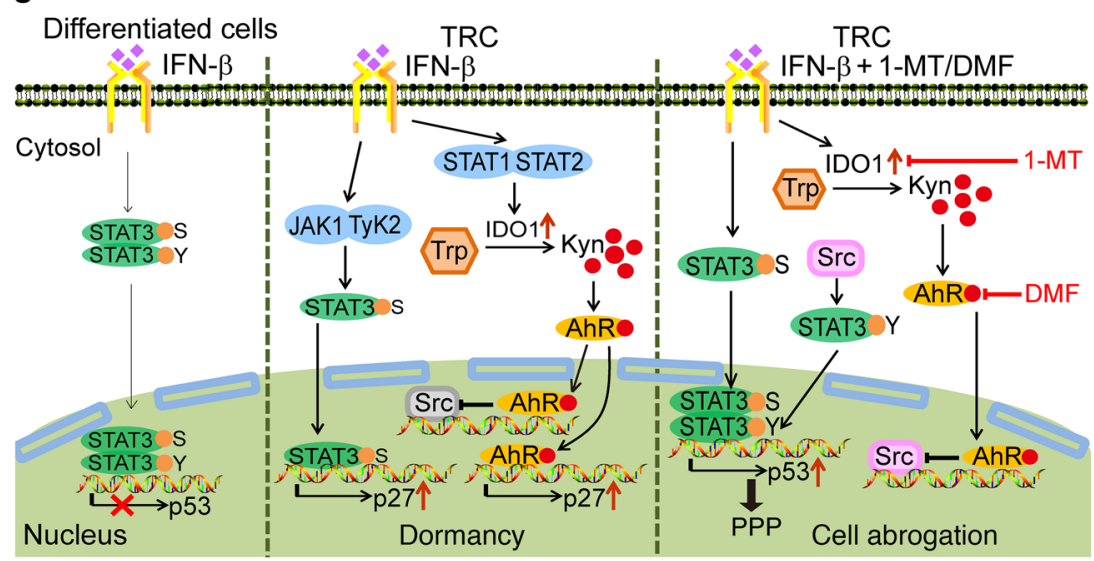


Figure 8. IDO/AhR blockade disrupts dormancy of primary human TRCs. (A) Primary human melanoma cells after 2 days culture in soft 3D fibrin gels were treated with IFN- $\beta(10 \mathrm{ng} / \mathrm{ml})$ for the indicated days $(n=3)$. (B and C) Primary human melanoma TRCs $(n=3)$ after 3 days IFN- $\beta$ treatment were subjected to cell-cycle analysis (B) or immunostaining of NR2F1 and Ki67 (C). (D) Same as B, but some cells were subjected to Western blot against ID01 and AhR (left) or immunostaining with anti-AhR (red) and DAPI (right) $(n=3)$. (E) NOD-SCID mice with $7 \times 7 \mathrm{~mm}$ melanoma by s.c. injection of primary human TRCs were treated with IFN- $\beta / 1-M T$ or IFN- $\beta$ / DMF for 10 days. Tumor growth was measured $(n=8)$. (F) IFN- $\beta$ expression in various human cancers from the NIH TCGA (The Cancer Cenome Atlas) database, including colon (638 TCGA entries), stomach (375 TCGA entries), breast (414 TCGA entries), urothelial (531 TCGA entries), lung (1033 TCGA entries), pancreatic (177 TCGA entries) cancers, and melanoma (472 TCGA entries), was analyzed according to the formula IFN $\beta=\log _{2}(F P K M+1)$, where FPKM indicates fragments per kb of transcript per million mapped reads. (G) Representatives of immunohistochemical staining IFN- $\beta$ from 10 melanoma patient samples (upper panels). Immunostaining intensity was quantified (lower panel) relative to the no. 1 patient, which was defined as 1. Patient samples with a calculated relative intensity greater than 10 were considered IFN- $\beta^{\text {hi }}$, while patient samples with a calculated relative intensity less than 10 were defined as IFN- $\beta^{\text {lo }}$. (H) Immunostaining of NR2F1 and Ki67 in IFN- $\beta^{\text {hi }}$ and IFN- $\beta^{\text {lo }}$ patients with melanoma $(n=2)$. The percentage of NR2F1+Ki67- or NR2F1-Ki67+ cells was calculated. (I) Correlation between IFN- $\beta$ expression and melanoma patients' survival. (J) Schematic diagram showing the signaling pathway involved in IFN- $\beta$-induced TRC dormancy. Data represent mean \pm SEM. ${ }^{* *} P<0.01$, by 2 -tailed Student's $t$ test $(\mathbf{A}-\mathbf{C}$, $\mathbf{G}$, and $\mathbf{H}), 1$-way ANOVA (E), and Kaplan-Meier survival analysis (I). Scale bars: $50 \mu \mathrm{m}$ (A and $\mathbf{C}) ; 10 \mu \mathrm{m}$ (C, D, and $\mathbf{H}$ ).

tion is why IFN- $\beta$ has such a dormancy-inducing function. It is reasonable to speculate that IFN- $\beta$, as a major innate host defense molecule, is selected to trigger virus-infected cells into dormancy as an antiviral strategy and that tumor cells share some features of virus-infected cells. Another question is why only TRCs, and not differentiated tumor cells, are susceptible to IFN- $\beta$-induced dormancy. This might be because dormancy is a hardwired program tightly associated with ancestral cells and stem-like cells, such as TRCs. The phenomenon of TRC dormancy is very useful for better understanding tumor cell behaviors and current tumor treatments. A marked feature of tumors lies in their heterogenicity (54). Stemlike tumor cells are responsible for tumor formation and their dormancy is involved in not only recurrence and metastasis, but also various types of treatment resistance (12). Linking tumor dormancy to stem-like tumor cells or TRCs would further provide insights into novel therapeutic strategies against tumor recurrence, metastasis, and treatment resistance. Although TRC dormancy can be induced by exogenous IFN- $\beta$, this could also be realized by endogenous IFN- $\beta$. Using a bioinformatic approach, we actually found that IFN- $\beta$ is widely expressed in tumor tissues, especially in breast cancer, which has the highest expression of IFN- $\beta$. This might be the reason why breast cancer cells bias to dormancy. Importantly, even if IFN- $\beta$ was not markedly expressed in melanoma samples of patients, we still found that high expression of IFN- $\beta$ is correlated with a dormant state of melanoma cells.

The signaling pathway of IFN- $\beta$ is well known by its activation of JAK1/TyK and STAT1/STAT2/IRF9 transcription factor complexes (8). However, in this study, we demonstrate a previously unknown aspect of the downstream process through which IFN- $\beta$ induces TRCs into dormancy. We found that IFN- $\beta$ activates an IDO1/ Kyn/AhR/p27 pathway to mediate TRC dormancy. In our previ- ous study, we found that IFN- $\gamma$ also uses the IDO1/Kyn/AhR/p27 pathway to induce TRC dormancy. However, compared with IFN- $\gamma$, IFN- $\beta$ seems to have a stronger capacity to induce TRC dormancy. The underlying molecular mechanism might be due to the different state of STAT3 phosphorylation. We found that IFN $-\gamma$ does not induce serine phosphorylation of STAT3. However, IFN- $\beta$ can lead to p-STAT3 (S). As a result, p-STAT3 (S) upregulates p27 expression by binding to the p27 promoter. This selective serine phosphorylation by IFN- $\beta$ may be caused either by Tyk, as IFN- $\gamma$ activates Jak1 and Jak2, while IFN- $\beta$ activates Jak1 and Tyk, or by Jak 2 , since a previous study shows that phosphorylation of Jak2 leads to the activation of STAT3 in a breast cancer model (55). Thus, IFN- $\beta$ can use p-STAT3 (S) to further enhance p27 expression, facilitating TRCs to enter dormancy. This serine phosphorylation is a very important molecular event in that it mediates the nuclear translocation of STAT3, which not only is commonly expressed, but is also highly phosphorylated in tumor cells and considered as an oncogene (56). As a cytoplasmic transcription factor, phosphorylation of STAT3 is required for its nuclear translocation. Tyrosine phosphorylation is the main form of STAT3 phosphorylation (57). However, studies have found that tyrosine phosphorylation is not necessary for STAT3 nuclear translocation (46). Notwithstanding tyrosine phosphorylation, in this study, we found that serine phosphorylation is required for STAT3 nuclear translocation. STAT3 in the nucleus was associated with serine phosphorylation. Furthermore, serine-mutated STAT3 lost its ability to enter the nucleus of TRCs. Although we identify serine phosphorylation as mediating the translocation of STAT3 into the nucleus, the detailed molecular mechanism of how serine phosphorylation influences the interaction of STAT3 with cytoskeletons and the nuclear pore remains unclear and is worthy of further study.

Serine phosphorylation not only mediates STAT3 entering the nucleus, but is also critical for STAT3-mediated gene regulation (56). In the present study, serine and tyrosine phosphorylation was found to be involved in regulating TRC dormancy. Activation of AhR by IFN- $\beta$ leads to decreased Src kinase activity and subsequently decreased tyrosine phosphorylation of STAT3. However, AhR does not interfere with serine phosphorylation induced by IFN- $\beta$. Thus, p-STAT3 (S) enters the nucleus where it binds the p27 promoter to upregulate p27 expression. On the other hand, AhR blockade liberates Src kinase activity, leading to the generation of p-STAT3 (S, Y). This dual phosphorylation form is also able to enter the nucleus, where it binds to the 553 promoter, but not the p27 promoter. This binding switch leads to the upregulation of p53 expression. A previous report showed that p53 induces tumor cell death by inhibiting GPDH, the enzyme catalyzing the first step of PPP (53), resulting in increased ROS. However, p53 has also been shown to exhibit an antioxidant function (58). Thus, the role of p53 in regulating the metabolism is complex, and controversy exists. In our settings, upregulated p53 was found to target the PPP. In addition to inhibiting GPDH, we additionally found that p53 actually strongly suppresses 6PGDH, which we believe has not been reported before. By real-time PCR, 6PGDH mRNA could almost not be detected, thus fully blocking PPP and resulting in a striking increase of ROS levels and subsequent TRC death. Based on the results reported here, we propose a mechanism by which IFN- $\beta$-induced dormant TRCs can be disrupted by AhR blockade (Figure 8E). The fact that the disruption of dormant TRCs is medi- 
ated through a p53-dependent shutdown of GPDH and 6PGDH might raise concern over p53 mutation. However, p53 mutation is mainly associated with advanced cancers and rarely occurs in early tumors (59). Furthermore, in the present study, we did not find that p53 mutation influences PPP. Thus, the p53-dependent dormancy abrogation seems to be an important mechanism, and whether a p53-independent pathway exists is worthy of further investigation. In line with this mechanism elucidation, we demonstrated that IFN- $\beta$ and $1-\mathrm{MT} / \mathrm{DMF}$ treatment inhibits human TRC-inoculated melanoma growth in a NOD-SCID mouse model. The magnitude of tumor growth inhibition might be improved in a bulk melanoma cell-inoculated mouse model because TRCs are more malignant and have a much higher proliferation capacity compared with the differentiated counterparts.

In summary, the data in this study clearly show that IFN- $\beta$, by virtue of its activating the IDO/Kyn/AhR cascade and serine phosphorylation of STAT3, induces dormancy of melanoma TRCs, leading to the discovery of the combination of IFN- $\beta$ and an IDO1 or AhR inhibitor for effectively abrogating dormant TRCs. These findings may open a new venue for cancer immunotherapy.

\section{Methods}

Study design. This controlled laboratory study was conducted to characterize the molecular circuitry behind immune-mediated tumor dormancy in tumor cell lines as well as primary human cancers and investigate the possibility of exploiting this pathway to induce dormancy-related apoptosis for cancer treatment.

Animal and cell lines. For the in vitro part of the study, mouse tumor cell lines B16 (melanoma) and H22 (hepatocellular carcinoma) and human tumor cell lines A375 (melanoma), MCF-7 and MDA-MB-231 (breast cancer), SGC-7901 (stomach cancer), and HEK-293T (human embryonic kidney) were purchased from China Center for Type Culture Collection and cultured in DMEM (Thermo Fisher Scientific) with $10 \%$ FBS (Gibco; Thermo Fisher Scientific), except that $\mathrm{H} 22$ cells were grown in RPMI 1640 medium (Gibco; Thermo Fisher Scientific) with $10 \%$ FBS. For the in vivo part of the study, 6- to 8-week-old female C57BL/6, BALB/c, and NOD-SCID mice were purchased from the Center of Medical Experimental Animals, Chinese Academy of Medical Sciences (Beijing, China). These animals were maintained in the Animal Facilities of the Chinese Academy of Medical Sciences under pathogen-free conditions. Experiments were repeated 3 times to ensure statistical significance. The sample size of mice $(n=5-10)$ was arbitrarily selected based on previous experience, thus indicating that this study was sufficiently powered. Investigators were not blinded in the animal treatment experiments. Resected human melanoma tissues were obtained from patients at the Peking Union Medical College.

Tumor cells cultured on a $2 \mathrm{D}$ rigid dish or in $3 D$ fibrin gels. For conventional $2 \mathrm{D}$ cell culture, tumor cells were maintained in a rigid dish with complete culture medium. TRC culture was performed according to our previously published protocol $(13,15,30)$ with some modification. In brief, fibrinogen (Searun Holdings Co.) was dissolved at $-4^{\circ} \mathrm{C}$ overnight and, once completely dissolved, was diluted into 2 $\mathrm{mg} / \mathrm{ml}$ with T7 buffer ( $\mathrm{pH}$ 7.4, $50 \mathrm{mM}$ Tris, $150 \mathrm{mM} \mathrm{NaCl}$ ). Then, a precooled mixture of fibrinogen and cell solution was seeded into a precooled 24 -well plate with $5 \mu \mathrm{l}$ thrombin $(0.1 \mathrm{U} / \mu \mathrm{l}$, Searun Holdings Co.) to let the mixture have enough time to mix with thrombin and form very homogenous 3D fibrin gels. Mixtures of $250 \mu \mathrm{l}$ cell/ fibrinogen were seeded into 24-well plates and mixed well with preadded $5 \mu$ lhrombin $(0.1 \mathrm{U} / \mu \mathrm{l}$, Searun Holdings Co.). After 30 minutes of incubation at $37^{\circ} \mathrm{C}$, these cells were supplemented with $1 \mathrm{ml}$ completed culture medium. In order to collect the cells cultured in soft 3D fibrin gels (90 Pascal), they were treated with dispase II (Roche, cata$\log 4942078001$ ) for 10 minutes at $37^{\circ} \mathrm{C}$.

Plasmids and reagents. pGFP-C-shLenti-shIDO1 and pLenti-CMyc-DDK-IDO1were purchased from Origene. pX330-p27-sgRNA, pX330-AhR-sgRNA, pX330-STAT1-sgRNA, pX330-STAT2-sgRNA, pX330-STAT3-sgRNA, pRc/CMV-Flag-STAT3, and pRc/CMV-FlagSTAT3-Y705F plasmids were purchased from Addgene. pRc/CMV-FlagSTAT3-S727A plasmid was constructed by making a single mutation from pRc/CMV-Flag-Stat3 plasmid. Kynurenine, 1-L-MT, methotrexate (MTX), paclitaxel (PAX), cisplatin, aspartame, and 3',4'-DMF were from Sigma-Aldrich. IFN- $\beta$ and anti-IFN- $\beta$ neutralized antibody were purchased from PeproTech. Salmon fibrinogen, thrombin, and dispase were from Reagent Proteins. Puromycin was from Invitrogen.

More information is available in Supplemental Experimental Procedures.

Statistics. All experiments were performed at least 3 times. To determine the colony size, at least 20 colonies from different fields were measured. Results are expressed as mean \pm SEM and were analyzed by Student's $t$ test (unpaired), 2-tailed, or 1-way ANOVA followed by Bonferroni's test. To analyze the correlation between IFN- $\beta$ expression and patient survival, Pearson's correlation test was applied. The survival rates were determined by Kaplan-Meier survival analysis. $P<0.05$ was considered statistically significant. The analysis was conducted using Graphpad 6.0 software. Sample exclusion was never carried out.

Study approval. All studies involving mice were approved by the Animal Care and Use Committee of the Chinese Academy of Medical Sciences. Melanoma tissues were obtained from patients at the Peking Union Medical College. Ethical permission was granted by the Clinical Trial Ethics Committee of Peking Union Medical College. All patients provided written, informed consent to participate in the study.

\section{Author contributions}

$\mathrm{BH}$ conceived the project. YL, J. Lv, J. Liu, XJ, XL, JX, LZ, DC, RF, KT, JM, Huafeng Zhang, WD, SM, TZ, YK, FC, YZ, and QJ performed the experiments. BH, YL, JG, Haizeng Zhang, XFQ, ZWH, and XC developed methodology. BH, YL, JL, QZ, BZ, XJ, XL, $\mathrm{ZWH}, \mathrm{XC}$, and $\mathrm{XFQ}$ performed data analyses. $\mathrm{BH}$ and $\mathrm{YL}$ wrote the manuscript with input from all authors.

\section{Acknowledgments}

This work was supported by the National Natural Science Foundation of China (81788101, 81661128007, 81530080, 81773062), the National Natural Science Fund for Young Scholars of China (81502473), and the Chinese Academy of Medical Sciences Initiative for Innovative Medicine (2016-I2M-1-007).

Address correspondence to: Bo Huang, National Key Laboratory of Medical Molecular Biology and Department of Immunology, Institute of Basic Medical Sciences, Clinical Immunology Center, Chinese Academy of Medical Sciences, Dongdansantiao No.5, Beijing 100005, China. Phone: 8610.6915.6447; Email: tjhuangbo@hotmail.com. 
1. Katze MG, He Y, Gale M. Viruses and interferon: a fight for supremacy. Nat Rev Immunol. 2002;2(9):675-687.

2 . Tjandra SS, et al. IFN- $\beta$ signaling positively regulates tumorigenesis in aggressive fibromatosis, potentially by modulating mesenchymal progenitors. Cancer Res. 2007;67(15):7124-7131.

3. Tschurtschenthaler M, et al. Type I interferon signalling in the intestinal epithelium affects Paneth cells, microbial ecology and epithelial regeneration. Gut. 2014;63(12):1921-1931.

4. Chen HM, et al. Critical role for constitutive type I interferon signaling in the prevention of cellular transformation. Cancer Sci. 2009;100(3):449-456.

5. Diamond MS, et al. Type I interferon is selectively required by dendritic cells for immune rejection of tumors. J Exp Med. 2011;208(10):1989-2003.

6. Fuertes MB, Woo S-R, Burnett B, Fu Y-X, Gajewski TF. Type I interferon response and innate immune sensing of cancer. Trends Immunol. 2013;34(2):67-73.

7. Einat M, Resnitzky D, Kimchi A. Close link between reduction of $\mathrm{c}$-myc expression by interferon and, GO/G1 arrest. Nature. 1985;313(6003):597-600.

8. Platanias LC. Mechanisms of type-I- and type-II-interferon-mediated signalling. Nat Rev Immunol. 2005;5(5):375-386.

9. MacKie RM, Reid R, Junor B. Fatal melanoma transferred in a donated kidney 16 years after melanoma surgery. $N$ Engl J Med. 2003;348(6):567-568.

10. Milton CA, Barbara J, Cooper J, Rao M, Russell C, Russ G. The transmission of donor-derived malignant melanoma to a renal allograft recipient. Clin Transplant. 2006;20(5):547-550.

11. Strauss DC, Thomas JM. Transmission of donor melanoma by organ transplantation. Lancet Oncol. 2010;11(8):790-796.

12. Sosa MS, Bragado P, Aguirre-Ghiso JA. Mechanisms of disseminated cancer cell dormancy: an awakening field. Nat Rev Cancer. 2014;14(9):611-622.

13. Liu J, et al. Soft fibrin gels promote selection and growth of tumourigenic cells. Nat Mater. 2012;11(8):734-741.

14. Tan Y, et al. Matrix softness regulates plasticity of tumour-repopulating cells via H3K9 demethylation and Sox2 expression. Nat Commun. 2014;5:4619.

15. Ma J, et al. Reversing drug resistance of soft tumor-repopulating cells by tumor cell-derived chemotherapeutic microparticles. Cell Res. 2016;26(6):713-727.

16. Li Y, et al. Upregulation of cytosolic phosphoenolpyruvate carboxykinase is a critical metabolic event in melanoma cells that repopulate tumors. Cancer Res. 2015;75(7):1191-1196.

17. Liu Y, et al. Blockade of IDO-kynurenine-AhR metabolic circuitry abrogates IFN- $\gamma$-induced immunologic dormancy of tumor-repopulating cells. Nat Commun. 2017;8:15207.

18. Eggermont AM, et al. Adjuvant therapy with pegylated interferon alfa- $2 \mathrm{~b}$ versus observation alone in resected stage III melanoma: final results of EORTC 18991, a randomised phase III trial. Lancet. 2008;372(9633):117-126.

19. Zitvogel L, Galluzzi L, Kepp O, Smyth MJ, Kroemer G. Type I interferons in anticancer immunity. Nat Rev Immunol. 2015;15(7):405-414.
20. Belardelli F, Ferrantini M, Proietti E, Kirkwood JM. Interferon-alpha in tumor immunity and immunotherapy. Cytokine Growth Factor Rev. 2002;13(2):119-134.

21. Pfeffer LM, et al. Biological properties of recombinant $\alpha$-interferons: 40th anniversary of the discovery of interferons. Cancer Res. 1998;58(12):2489-2499.

22. Kusumbe AP, Bapat SA. Cancer stem cells and aneuploid populations within developing tumors are the major determinants of tumor dormancy. Cancer Res. 2009;69(24):9245-9253.

23. Kobayashi A, et al. Bone morphogenetic protein 7 in dormancy and metastasis of prostate cancer stem-like cells in bone. J Exp Med. 2011;208(13):2641-2655.

24. Kleffel S, Schatton T. Tumor dormancy and cancer stem cells: two sides of the same coin? In: Enderling H, Almog N, Hlatky L, eds. Systems Biology of Tumor Dormancy. New York, New York, USA: Springer; 2013:145-179.

25. Cheung TH, Rando TA. Molecular regulation of stem cell quiescence. Nat Rev Mol Cell Biol. 2013;14(6):329-340.

26. Zimmerer RM, et al. Functional features of cancer stem cells in melanoma cell lines. Cancer Cel Int. 2013;13(1):78.

27. Monzani E, et al. Melanoma contains CD133 and ABCG2 positive cells with enhanced tumourigenic potential. Eur J Cancer. 2007;43(5):935-946.

28. Fluegen $G$, et al. Phenotypic heterogeneity of disseminated tumour cells is preset by primary tumour hypoxic microenvironments. Nat Cell Biol. 2017;19(2):120-132.

29. Sosa MS, et al. NR2F1 controls tumour cell dormancy via SOX9- and RAR $\beta$-driven quiescence programmes. Nat Commun. 2015;6:6170.

30. Liu Y, et al. Blockade of IDO-kynurenine-AhR metabolic circuitry abrogates IF $\mathrm{N}-\boldsymbol{\gamma}$-induced immunologic dormancy of tumor-repopulating cells. Nat Commun. 2017;8:15207.

31. Goss PE, Chambers AF. Does tumour dormancy offer a therapeutic target? Nat Rev Cancer. 2010;10(12):871-877.

32. Ghajar CM. Metastasis prevention by targeting the dormant niche. Nat Rev Cancer. 2015;15(4):238-247.

33. Takikawa O, Habara-Ohkubo A, Yoshida R. IFN- $\gamma$ is the inducer of indoleamine 2,3-dioxygenase in allografted tumor cells undergoing rejection. J Immunol. 1990;145(4):1246-1250.

34. Hosseini-Tabatabaei A, Jalili RB, Li Y, Kilani RT, Moeen Rezakhanlou A, Ghahary A. Mechanism underlying defective interferon gamma-induced IDO expression in non-obese diabetic mouse fibroblasts. PLoS One. 2012;7(5):e37747.

35. Opitz CA, et al. An endogenous tumourpromoting ligand of the human aryl hydrocarbon receptor. Nature. 2011;478(7368):197-203.

36. Nukaya M, Moran S, Bradfield CA. The role of the dioxin-responsive element cluster between the Cyp1a1 and Cyp1a2 loci in aryl hydrocarbon receptor biology. Proc Natl Acad Sci US A. 2009;106(12):4923-4928.

37. Gamero AM, et al. Activation of Tyk 2 and Stat 3 is required for the apoptotic actions of interferon- $\beta$ in primary pro-B cells. J Biol Chem . 2006;281(24):16238-16244
38. Tanabe Y, Nishibori T, Su L, Arduini RM, Baker DP, David M. Cutting edge: role of STAT1, STAT3, and STAT 5 in IFN- $\alpha \beta$ responses in T lymphocytes. JImmunol. 2005;174(2):609-613.

39. Yang CH, Murti A, Pfeffer LM. STAT3 complements defects in an interferon-resistant cell line: evidence for an essential role for STAT3 in interferon signaling and biological activities. Proc Natl Acad Sci U S A. 1998;95(10):5568-5572.

40. Wang WB, Levy DE, Lee CK. STAT3 negatively regulates type I IFN-mediated antiviral response. J Immunol. 2011;187(5):2578-2585.

41. Germain D, Frank DA. Targeting the cytoplasmic and nuclear functions of signal transducers and activators of transcription 3 for cancer therapy. Clin Cancer Res. 2007;13(19):5665-5669.

42. Aggarwal BB, et al. Signal transducer and activator of transcription-3, inflammation, and cancer: how intimate is the relationship? Ann N Y Acad Sci. 2009;1171:59-76.

43. He G, Karin M. NF-кB and STAT3 - key players in liver inflammation and cancer. Cell Res. 2011;21(1):159-168.

44. Buchert M, Burns CJ, Ernst M. Targeting JAK kinase in solid tumors: emerging opportunities and challenges. Oncogene. 2016;35(8):939-951.

45. Levy DE, Darnell JE. Stats: transcriptional control and biological impact. Nat Rev Mol Cell Biol. 2002;3(9):651-662.

46. Liu L, McBride KM, Reich NC. STAT3 nuclear import is independent of tyrosine phosphorylation and mediated by importin-alpha3. Proc Natl Acad Sci U S A. 2005;102(23):8150-8155.

47. Vogelstein B, Lane D, Levine AJ. Surfing the p53 network. Nature. 2000;408(6810):307-310.

48. Vousden KH, Lu X. Live or let die: the cell's response to p53. Nat Rev Cancer. 2002;2(8):594-604.

49. Yu H, et al. LIF negatively regulates tumour-suppressor p53 through Stat3/ID1/MDM2 in colorectal cancers. Nat Commun. 2014;5:5218.

50. Niu G, et al. Role of Stat 3 in regulating p53 expression and function. Mol Cell Biol. 2005;25(17):7432-7440.

51. Maillet A, Pervaiz S. Redox regulation of p53, redox effectors regulated by p53: a subtle balance. Antioxid Redox Signal. 2012;16(11):1285-1294.

52. He Z, Simon HU. A novel link between p53 and ROS. Cell Cycle. 2013;12(2):201-202.

53. Jiang $\mathrm{P}$, et al. p53 regulates biosynthesis through direct inactivation of glucose-6-phosphate dehydrogenase. Nat Cell Biol. 2011;13(3):310-316.

54. Hanahan D, Weinberg RA. Hallmarks of cancer: the next generation. Cell. 2011;144(5):646-674.

55 . Gao H, et al. Multi-organ site metastatic reactivation mediated by non-canonical discoidin domain receptor 1 signaling. Cell. 2016;166(1):47-62.

56. Decker T, Kovarik P. Serine phosphorylation of STATs. Oncogene. 2000;19(21):2628-2637.

57. Stark GR, Darnell JE. The JAK-STAT pathway at twenty. Immunity. 2012;36(4):503-514.

58. Sablina AA, Budanov AV, Ilyinskaya GV, Agapova LS, Kravchenko JE, Chumakov PM. The antioxidant function of the p53 tumor suppressor. Nat Med. 2005;11(12):1306-1313.

59. Kandoth C, et al. Mutational landscape and significance across 12 major cancer types. Nature. 2013;502(7471):333-339. 